\title{
Research Article \\ On the Necessary and Sufficient Condition for a Set of Matrices to Commute and Some Further Linked Results
}

\author{
M. De La Sen \\ Department of Electricity and Electronics, Faculty of Science and Technology, \\ University of the Basque Country, Campus of Leioa (Bizkaia). Aptdo. 644, 48080 Bilbao, Spain \\ Correspondence should be addressed to M. De La Sen, manuel.delasen@ehu.es \\ Received 5 January 2009; Revised 4 May 2009; Accepted 9 June 2009 \\ Recommended by Angelo Luongo
}

This paper investigates the necessary and sufficient condition for a set of (real or complex) matrices to commute. It is proved that the commutator $[A, B]=0$ for two matrices $A$ and $B$ if and only if a vector $v(B)$ defined uniquely from the matrix $B$ is in the null space of a well-structured matrix defined as the Kronecker sum $A \oplus\left(-A^{*}\right)$, which is always rank defective. This result is extendable directly to any countable set of commuting matrices. Complementary results are derived concerning the commutators of certain matrices with functions of matrices $f(A)$ which extend the well-known sufficiency-type commuting result $[A, f(A)]=0$.

Copyright $@ 2009$ M. De La Sen. This is an open access article distributed under the Creative Commons Attribution License, which permits unrestricted use, distribution, and reproduction in any medium, provided the original work is properly cited.

\section{Introduction}

The problem of commuting operators and matrices, in particular, is very relevant in a significant number of problems of several branches of science, which are very often mutually linked, cited herein after.

(1) In several fields of interest in Applied Mathematics or Linear Algebra [1-22] including Fourier transform theory, graph theory where, for instance, the commutativity of the adjacency matrices is relevant [1, 17-19, 21-35], Lyapunov stability theory with conditional and unconditional stability of switched dynamic systems involving discrete systems, delayed systems, and hybrid systems where there is a wide class of topics covered including their corresponding adaptive versions including estimation schemes (see, e.g., [2341]). Generally speaking, linear operators, and in particular matrices, which commute share some common eigenspaces. On the other hand, a known mathematical result is that two graphs with the same vertex set commute if their adjacency matrices commute [16]. Graphs are abstract representations of sets of objects (vertices) where some pairs of them are connected by links (arcs/edges). Graphs are often used to describe behaviors of multiconfiguration switched 
systems where nodes represent each parameterized dynamics and arcs describe allowed switching transitions [35]. They are also used to describe automatons in Computer Science. Also, it has been proven that equalities of products involving two linear combinations of two any length products having orthogonal projectors (i.e., Hermitian idempotent matrices) as factors are equivalent to a commutation property [21].

(2) In some fields in Engineering, such as multimodel regulation and Parallel multiestimation [36-41]. Generally speaking switching among configurations can improve the transient behavior. Switching can be performed arbitrarily (i.e., at any time instant) through time while guaranteeing closed-loop stability if a subset of the set of configurations is stable provided that a common Lyapunov function exists for them. This property is directly related to certain pair wise commutators of matrices describing configuration dynamics being zero [7, 10, 11, 14, 15]. Thus, the problem of commuting matrices is in fact of relevant interest in dynamic switched systems, namely, those which possess several parameterized configurations, one of them, is becoming active at each current time interval. If the matrices of dynamics of all the parameterizations commute then there exists a common Lyapunov function for all those parameterizations and any arbitrary switching rule operating at any time instant maintains the global stability of the switched rule provided that all the parameterizations are stable [7]. This property has been described also in [23-25, 28-30] and many other references therein. In particular, there are recent studies which prove that, in these circumstances, arbitrary switching is possible if the matrices of dynamics of the various configurations commute while guaranteeing closed-loop stability. This principle holds not only in both the continuous-time delay-free case and in the discrete-time one but even in configurations involving time-delay and hybrid systems as well. See, for instance, [10-15, 27-30, 34-41] and references therein. The set of involved problems is wide enough like, for instance, switched multimodel techniques [27-30, 35, 36, 40, 41], switched multiestimation techniques with incorporated parallel multiestimation schemes involving adaptive control [34, 3840], time delay and hybrid systems with several configurations under mutual switching, and so forth $[10,11,14,15]$ and references therein. Multimodel tools and their adaptive versions incorporating parallel multiestimation are useful to improve the regulation and tracking transients including those related to triggering circuits with regulated transient via multiestimation [36], master-slave tandems [39], and so forth. However, it often happens that there is no common Lyapunov function for all the parameterizations becoming active at certain time intervals. Then, a minimum residence (or dwelling) time at each active parameterization has to be respected before performing the next switching in order to guarantee the global stability of the whole switched system so that the switching rule among distinct parameterizations is not arbitrary $[7,12,13,27-30,34-41]$.

(3) In some problems of Signal Processing. See, for instance, $[1,17,18]$ concerning the construction of DFT (Discrete Fourier transform)-commuting matrices. In particular, a complete orthogonal set of eigenvectors can be obtained for several types of offset DFT's and DCT's under commutation properties.

(4) In certain areas of Physics, and in particular, in problems related to Quantum Mechanics. See, for instance, [22, 42,43]. Basically, a complete set of commuting observables is a set of commuting operators whose eigenvalues completely specify the state of a system since they share eigenvectors and can be simultaneously measured [22, 42, 43]. These Quantum Mechanics tools have also inspired other Science branches. For instance, it is investigated in the above mentioned reference [18] a commuting matrix whose eigenvalue spectrum is very close to that of the Gauss-Hermite differential operator. It is proven that it furnishes two generators of the group of matrices which commute with the discrete Fourier transform. It is also pointed out 
that the associate research inspired in Quantum Mechanics principles. There is also other relevant basic scientific applications of commuting operators. For instance, the symmetry operators in the point group of a molecule always commute with its Hamiltonian operator [20]. The problem of commuting matrices is also relevant to analyze the normal modes in dynamic systems or the discussion of commuting matrices dependent on a parameter (see, e.g., [2,3]).

It is well known that commuting matrices have at least a common eigenvector and also, a common generalized eigenspace $[4,5]$. A less restrictive problem of interest in the above context is that of almost commuting matrices, roughly speaking, the norm of the commutator is sufficiently small $[5,6]$. A very relevant related result is that the sum of matrices which commute is an infinitesimal generator of a $C_{0}$-semigroup. This leads to a wellknown result in Systems Theory establishing that the matrix function $e^{A_{1} t_{1}+A_{2} t_{2}}=e^{A_{1} t_{1}} e^{A_{2} t_{2}}$ is a fundamental (or state transition) matrix for the cascade of the time invariant differential systems $\dot{x}_{1}(t)=A_{1} x_{1}(t)$, operating on a time $t_{1}$, and $\dot{x}_{2}(t)=A_{2} x_{2}(t)$, operating on a time $t_{2}$, provided that $A_{1}$ and $A_{2}$ commute (see, e.g., [7-11]).

Most of the abundant existing researches concerning sets of commuting operators, in general, and matrices, in particular, are based on the assumption of the existence of such sets implying that each pair of mutual commutators is zero. There is a gap in giving complete conditions guaranteeing that such commutators within the target set are zero. This paper formulates the necessary and sufficient condition for any countable set of (real or complex) matrices to commute. The sequence of obtained results is as follows. Firstly, the commutation of two real matrices is investigated in Section 2. The necessary and sufficient condition for two matrices to commute is that a vector defined uniquely from the entries of any of the two given matrices belongs to the null space of the Kronecker sum of the other matrix and its minus transpose. The above result allows a simple algebraic characterization and computation of the set of commuting matrices with a given one. It also exhibits counterparts for the necessary and sufficient condition for two matrices not to commute. The results are then extended to the necessary and sufficient condition for commutation of any set of real matrices in Section 3. In Section 4, the previous results are directly extended to the case of complex matrices in two very simple ways, namely, either by decomposing the associated algebraic system of complex matrices into two real ones or by manipulating it directly as a complex algebraic system of equations. Basically, the results for the real case are directly extendable by replacing transposes by conjugate transposes. Finally, further results concerning the commutators of matrices with matrix functions are also discussed in Section 4. The proofs of the main results in Sections 2, 3, and 4 are given in corresponding Appendices A, B, and C. It may be pointed out that there is implicit following duality of the main result. Since a necessary and sufficient condition for a set of matrices to commute is formulated and proven, the necessary and sufficient condition for a set of matrices not to commute is just the failure in the above one to hold.

\subsection{Notation}

$[A, B]$ is the commutator of the square matrices $A$ and $B$. $A \otimes B:=\left(a_{i j} B\right)$ is the Kronecker (or direct) product of $A:=\left(a_{i j}\right)$ and $B$.

$A \oplus B:=A \otimes I_{n}+I_{n} \otimes B$ is the Kronecker sum of the square matrices $A:=\left(a_{i j}\right)$ and both of order $n$, where $I_{n}$ is the $n$th identity matrix.

$A^{T}$ is the transpose of the matrix $A$ and $A^{*}$ is the conjugate transpose of the complex matrix $A$. For any matrix $A, \operatorname{Im} A$ and $\operatorname{Ker} A$ are its associate range (or image) subspace and 
null space, respectively. Also, $\operatorname{rank}(A)$ is the rank of $A$ which is the dimension of $\operatorname{Im}(A)$ and $\operatorname{det}(A)$ is the determinant of the square matrix $A$. matrix $A$.

$v(A)=\left(a_{1}^{T}, a_{2}^{T}, \ldots, a_{n}^{T}\right)^{T} \in \mathrm{C}^{n^{2}}$ if $a_{i}^{T}:=\left(a_{i 1}, a_{i 2}, \ldots, a_{i n}\right)$ is the $i$ th row of the square

$\sigma(A)$ is the spectrum of $A ; \bar{n}:=\{1,2, \ldots, n\}$. If $\lambda_{i} \in \sigma(A)$ then there exist positive integers $\mu_{i}$ and $v_{i} \leq \mu_{i}$ which are, respectively, its algebraic and geometric multiplicity; that is, the times it is repeated in the characteristic polynomial of $A$ and the number of its associate Jordan blocks, respectively. The integer $\mu \leq n$ is the number of distinct eigenvalues and the integer $m_{i}$, subject to $1 \leq m_{i} \leq \mu_{i}$, is the index of $\lambda_{i} \in \sigma(A) ; \forall i \in \bar{\mu}$, that is, its multiplicity in the minimal polynomial of $A$.

$A \sim B$ denotes a similarity transformation from $A$ to $B=T^{-1} A T$ for given $A, B \in$ $\mathbf{R}^{n \times n}$ for some nonsingular $T \in \mathbf{R}^{n \times n} . A \approx B=E A F$ means that there is an equivalence transformation for given $A, B \in \mathbf{R}^{n \times n}$ for some nonsingular $E, F \in \mathbf{R}^{n \times n}$.

A linear transformation from $\mathbf{R}^{n}$ to $\mathbf{R}^{n}$, represented by the matrix $T \in \mathbf{R}^{n \times n}$, is denoted identically to such a matrix in order to simplify the notation. If $V \neq \operatorname{Dom} T \equiv \mathbf{R}^{n}$ is a subspace of $\mathbf{R}^{n}$ then $\operatorname{Im} T(V):=\{T z: z \in V\}$ and $\operatorname{Ker} T(V):=\left\{z \in V: T z=0 \in \mathbf{R}^{n}\right\}$. If $V \equiv \mathbf{R}^{n}$, the notation is simplified to $\operatorname{Im} T:=\left\{T z: z \in \mathbf{R}^{n}\right\}$ and $\operatorname{Ker} T:=\left\{z \in \mathbf{R}^{n}: T z=0 \in \mathbf{R}^{n}\right\}$.

The symbols " $\wedge$ " and " $\vee$ " stand for logic conjunction and disjunction, respectively. The abbreviation "iff" stands for "if and only if." The notation card $U$ stands for the cardinal of the set $U . C_{A}$ (resp., $\bar{C}_{A}$ ) is the set of matrices which commute (resp., do not commute) with a matrix $A . C_{\mathrm{A}}$ (resp., $\bar{C}_{\mathrm{A}}$ ) is the set of matrices which commute (resp., do not commute) with all square matrix $A_{i}$ belonging to a given set $\mathbf{A}$.

\section{Results Concerning the Sets of Commuting and No Commuting Matrices with a Given One}

Consider the sets $C_{A}:=\left\{X \in \mathbf{R}^{n \times n}:[A, X]=0\right\} \neq \emptyset$, of matrices which commute with $A$, and $\bar{C}_{A}:=\left\{X \in \mathbf{R}^{n \times n}:[A, X] \neq 0\right\}$, of matrices which do not commute with $A ; \forall A \in \mathbf{R}^{n \times n}$. Note that $0 \in \mathbf{R}^{n \times n} \cap C_{A}$; that is, the zero $n$-matrix commutes with any $n$-matrix so that, equivalently, $0 \notin \mathbf{R}^{n \times n} \cap \bar{C}_{A}$ and then $C_{A} \cap \bar{C}_{A}=\emptyset ; \forall A \in \mathbf{R}^{n \times n}$. The subsequent two basic results which follow are concerned with commutation and noncommutation of two real matrices $A$ and $X$. The used tool relies on the calculation of the null space and the range space of the Kronecker sum of the matrix $A$, one of the matrices, with its minus transpose. A vector built with all the entries of the other matrix $X$ has to belong to one of the above spaces for $A$ and $X$ to commute and to the other one in order that $A$ and $X$ not to be two commuting matrices.

Proposition 2.1. (i) $C_{A}=\left\{X \in \mathbf{R}^{n \times n}: v(X) \in \operatorname{Ker}\left(A \oplus\left(-A^{T}\right)\right)\right\}$.

(ii) $\bar{C}_{A}=\mathbf{R}^{n \times n} \backslash C_{A}=\left\{X \in \mathbf{R}^{n \times n}: v(X) \notin \operatorname{Ker}\left(A \oplus\left(-A^{T}\right)\right)\right\} \equiv\left\{X \in \mathbf{R}^{n \times n}: v(X) \in\right.$ $\left.\operatorname{Im}\left(A \oplus\left(-A^{T}\right)\right)\right\}$.

(iii) $B \in C_{A}:=\left\{X \in \mathbf{R}^{n \times n}: v(X) \in \operatorname{Ker}\left(A \oplus\left(-A^{T}\right)\right)\right\}$.

Note that according to Proposition 2.1 the set of matrices $C_{A}$ which commute with the square matrix $A$ and its complementary $\bar{C}_{A}$ (i.e., the set of matrices which do not commute with $A$ ) can be redefined in an equivalent way by using their given expanded vector forms. 
Proposition 2.2. One has

$$
\begin{aligned}
\operatorname{rank}\left(A \oplus\left(-A^{T}\right)\right)<n^{2} & \Longleftrightarrow \operatorname{Ker}\left(A \oplus\left(-A^{T}\right)\right) \neq 0 \\
& \Longleftrightarrow 0 \in \sigma\left(A \oplus\left(-A^{T}\right)\right) \\
& \Longleftrightarrow \exists X(\neq 0) \in C_{A}, \quad \forall A \in \mathbf{R}^{n \times n} .
\end{aligned}
$$

Proof. One has $[A, A]=0 ; \forall A \in \mathbf{R}^{n \times n} \Rightarrow \exists \mathbf{R}^{n^{2}} \ni 0 \neq v(A) \in \operatorname{Ker}\left(A \oplus\left(-A^{T}\right)\right) ; \forall A \in \mathbf{R}^{n \times n}$. As a result,

$$
\operatorname{Ker}\left(A \oplus\left(-A^{T}\right)\right) \neq 0 \in \mathbf{R}^{n^{2}} ; \quad \forall A \in \mathbf{R}^{n \times n} \Longleftrightarrow \operatorname{rank}\left(A \oplus\left(-A^{T}\right)\right)<n^{2} ; \quad \forall A \in \mathbf{R}^{n \times n}
$$

so that $0 \in \sigma\left(A \oplus\left(-A^{T}\right)\right)$.

Also, $\exists X(\neq 0) \in \mathbf{R}^{n \times n}:[A, X]=0 \Leftrightarrow X \in C_{A}$ since $\operatorname{Ker}\left(A \oplus\left(-A^{T}\right)\right) \neq 0 \in \mathbf{R}^{n^{2}}$.

Then, Proposition 2.2 has been proved.

The subsequent mathematical result is stronger than Proposition 2.2 and is based on characterization of the spectrum and eigenspaces of $A \oplus\left(-A^{T}\right)$.

Theorem 2.3. The following properties hold.

(i) The spectrum of $A \oplus\left(-A^{T}\right)$ is $\sigma\left(A \oplus\left(-A^{T}\right)\right)=\left\{\bar{\lambda}_{i j}=\lambda_{i}-\lambda_{j}: \lambda_{i}, \lambda_{j} \in \sigma(A) ; \forall i, j \in \bar{n}\right\}$ and possesses $\bar{v}$ Jordan blocks in its Jordan canonical form of, subject to the constraints $n^{2} \geq \bar{v}=$ $\operatorname{dim} S=\left(\sum_{i=1}^{\mu} v_{i}\right)^{2} \geq \bar{v}(0)$, and $0 \in \sigma\left(A \oplus\left(-A^{T}\right)\right)$ with an algebraic multiplicity $\bar{\mu}(0)$ and with a geometric multiplicity $\bar{v}(0)$ subject to the constraints:

$$
n^{2}=\left(\sum_{i=1}^{\mu} \mu_{i}\right)^{2} \geq \bar{\mu}(0) \geq \sum_{i=1}^{\mu} \mu_{i}^{2} \geq \bar{v}(0)=\sum_{i=1}^{\mu} v_{i}^{2} \geq n,
$$

where

(a) $S:=\operatorname{span}\left\{z_{i} \otimes x_{j}, \forall i, j \in \bar{n}\right\}, \mu_{i}=\mu\left(\lambda_{i}\right)$ and $v_{i}=v\left(\lambda_{i}\right)$ are, respectively, the algebraic and the geometric multiplicities of $\lambda_{i} \in \sigma(A), \forall i \in \bar{n} ; \mu \leq n$ is the number of distinct $\lambda_{i} \in \sigma(A)(i \in \mu), \bar{\mu}_{i}=\bar{\mu}\left(\bar{\lambda}_{i j}\right)$ and $\bar{v}_{i j}=\bar{v}\left(\bar{\lambda}_{i j}\right)$, are, respectively, the algebraic and the geometric multiplicity of $\bar{\lambda}_{i j}=\left(\lambda_{i}-\lambda_{j}\right) \in \sigma\left(A \oplus\left(-A^{T}\right)\right), \forall i, j \in \bar{n} ; \mu \leq n$,

(b) $x_{j}$ and $z_{i}$ are, respectively, the right eigenvectors of $A$ and $A^{T}$ with respective associated eigenvalues $\lambda_{j}$ and $\lambda_{i} ; \forall i, j \in \bar{n}$.

(ii) One has

$$
\begin{aligned}
\operatorname{dim} \operatorname{Im}\left(A \oplus\left(-A^{T}\right)\right) & =\operatorname{rank}\left(A \oplus\left(-A^{T}\right)\right) \\
& =n^{2}-\bar{v}(0) \Longleftrightarrow \operatorname{dim} \operatorname{Ker}\left(A \oplus\left(-A^{T}\right)\right)=\bar{v}(0) ; \quad \forall A \in \mathbf{R}^{n \times n}
\end{aligned}
$$


Expressions which calculate the sets of matrices which commute and which do not commute with a given one are obtained in the subsequent result.

Theorem 2.4. The following properties hold.

(i) One has

$$
\begin{aligned}
X \in C_{A} \quad \text { iff }\left(A \oplus\left(-A^{T}\right)\right) v(X)=0 \\
\qquad X \in C_{A} \quad \text { iff } v(X)=-F\left(v^{T}\left(\bar{X}_{2}\right) \bar{A}_{12}^{T} \bar{A}_{11}^{-T}, v^{T}\left(\bar{X}_{2}\right)\right)^{T}
\end{aligned}
$$

for any $v\left(\overline{\mathrm{X}}_{2}\right) \in \operatorname{Ker}\left(\bar{A}_{22}-\bar{A}_{21} \bar{A}_{11}^{-1} \bar{A}_{12}\right)$, where $E, F \in \mathbf{R}^{n^{2} \times n^{2}}$ are permutation matrices and $\overline{\mathrm{X}} \in \mathbf{R}^{n \times n}$ and $v(\bar{X}) \in \mathbf{R}^{n^{2}}$ are defined as follows.

(a) One has

$$
v(\bar{X}):=F^{-1} v(X), \quad A \oplus\left(-A^{T}\right) \approx \bar{A}:=E\left(A \oplus\left(-A^{T}\right)\right) F, \quad \forall X \in C_{A},
$$

where $v(\bar{X})=\left(v^{T}\left(\bar{X}_{1}\right), v^{T}\left(\bar{X}_{2}\right)\right)^{T} \in \mathbf{R}^{n^{2}}$ with $v\left(\bar{X}_{1}\right) \in \mathbf{R}^{\bar{v}(0)}$ and $v\left(\bar{X}_{2}\right) \in \mathbf{R}^{n^{2}-\bar{v}(0)}$.

(b) The matrix $\bar{A}_{11} \in \mathbf{R}^{\bar{v}(0) \times \bar{v}(0)}$ is nonsingular in the block matrix partition $\bar{A}:=$ Block matrix $\left(\bar{A}_{i j} ; i, j \in \overline{2}\right)$ with $\bar{A}_{12} \in \mathbf{R}^{\overline{\bar{v}}(0) \times n^{2}}, \bar{A}_{21} \in \mathbf{R}^{\left(n^{2}-\bar{v}(0)\right) \times \bar{v}(0)}$ and $\bar{A}_{22} \in$ $\mathbf{R}^{\left(n^{2}-\bar{v}(0)\right) \times\left(n^{2}-\bar{v}(0)\right)}$.

(ii) $X \in \bar{C}_{A}$, for any given $A(\neq 0) \in \mathbf{R}^{n \times n}$, if and only if

$$
\left(A \oplus\left(-A^{T}\right)\right) v(X)=v(M)
$$

for some $M(\neq 0) \in \mathbf{R}^{n \times n}$ such that

$$
\operatorname{rank}\left(A \oplus\left(-A^{T}\right)\right)=\operatorname{rank}\left(A \oplus\left(-A^{T}\right), v(M)\right)=n^{2}-\bar{v}(0)
$$

Also,

$$
\begin{gathered}
\bar{C}_{A}:=\left\{X \in \mathbf{R}^{n \times n}:\left(A \oplus\left(-A^{T}\right)\right) v(X)=v(M) \text { for any } M(\neq 0) \in \mathbf{R}^{n \times n}\right. \text { satisfying } \\
\left.\operatorname{rank}\left(A \oplus\left(-A^{T}\right)\right)=\operatorname{rank}\left(A \oplus\left(-A^{T}\right), v(M)\right)=n^{2}-\bar{v}(0)\right\}
\end{gathered}
$$

Also, with the same definitions of $E, F$, and $\bar{X}$ in (i), $X \in \bar{C}_{A}$ if and only if

$$
v(X)=F\left(v^{T}\left(\bar{M}_{1}\right) \bar{A}_{11}^{-T}-v^{T}\left(\bar{X}_{2}\right) \bar{A}_{12}^{T} \bar{A}_{11}^{-T}, v^{T}\left(\bar{X}_{2}\right)\right)^{T}
$$


where $v\left(\bar{X}_{2}\right)$ is any solution of the compatible algebraic system

$$
\left(\bar{A}_{22}-\bar{A}_{21} \bar{A}_{11}^{-1} \bar{A}_{12}\right) v\left(\bar{X}_{2}\right)=v\left(\bar{M}_{2}\right)-\bar{A}_{21} \bar{A}_{11}^{-1} v\left(\bar{M}_{1}\right)
$$

for some $M(\neq 0) \in \mathbf{R}^{n \times n}$ such that $\bar{X}, \bar{M} \in \mathbf{R}^{n \times n}$ which are defined according to $v(X)=F v(\bar{X})$ and $\bar{M}=E M F \approx M(\neq 0) \in \mathbf{R}^{n \times n}$ with $v(\bar{M})=E v(M)=E\left(v_{1}^{T}(M), v_{2}^{T}(M)\right)^{T}$.

\section{Results Concerning Sets of Pair Wise Commuting Matrices}

Consider the following sets.

(1) A set of nonzero $p \geq 2$ distinct pair wise commuting matrices $\mathbf{A}_{C}:=\left\{A_{i} \in \mathbf{R}^{n \times n}\right.$ : $\left.\left[A_{i}, A_{j}\right]=0 ; \forall i, j \in \bar{p}\right\}$.

(2) The set of matrices $\mathrm{MC}_{\mathbf{A}_{C}}:=\left\{X \in \mathbf{R}^{n \times n}:\left[X, A_{i}\right]=0 ; \forall A_{i} \in \mathbf{A}_{C}\right\}$ which commute with the set $\mathbf{A}_{C}$ of pair wise commuting matrices.

(3) A set of matrices $C_{\mathbf{A}}:=\left\{X \in \mathbf{R}^{n \times n}:\left[X, A_{i}\right]=0 ; \forall A_{i} \in \mathbf{A}\right\}$ which commute with a given set of nonzero $p$ matrices $\mathbf{A}:=\left\{A_{i} \in \mathbf{R}^{n \times n} ; \forall i \in \bar{p}\right\}$ which are not necessarily pair wise commuting.

The complementary sets of $\mathrm{MC}_{\mathrm{A}_{C}}$ and $C_{\mathrm{A}}$ are $\overline{\mathrm{MC}_{\mathbf{A}_{C}}}$ and $\overline{C_{\mathrm{A}}}$, respectively, so that $\mathbf{R}^{n \times n} \ni B \in$ $\overline{\mathrm{MC}_{\mathbf{A}_{C}}}$ if $B \notin \mathrm{MC}_{\mathbf{A}_{C}}$ and $\mathbf{R}^{n \times n} \ni B \in \overline{C_{\mathbf{A}}}$ if $B \notin C_{\mathbf{A}}$. Note that $C_{\mathbf{A}_{C}}=\mathrm{MC}_{A_{C}}$ for a set of pair wise commuting matrices $\mathbf{A}_{C}$ so that the notation $\mathrm{MC}_{A_{C}}$ is directly referred to a set of matrices which commute with all those in a set of pair wise commuting matrices. The following two basic results are concerned with the commutation and noncommutation properties of two matrices.

Proposition 3.1. The following properties hold.

(i) One has

$$
\begin{aligned}
A_{i} \in \mathbf{A}_{C} ; \quad \forall i \in \bar{p} & \Longleftrightarrow v\left(A_{i}\right) \in \bigcap_{j(\neq i) \in \bar{p}} \operatorname{Ker}\left(A_{j} \oplus\left(-A_{j}^{T}\right)\right) ; \quad \forall i \in \bar{p} \\
& \Longleftrightarrow v\left(A_{i}\right) \in \bigcap_{i+1 \leq j \leq p} \operatorname{Ker}\left(A_{j} \oplus\left(-A_{j}^{T}\right)\right) ; \quad \forall i \in \bar{p} .
\end{aligned}
$$

(ii) Define

$$
\begin{aligned}
& N_{i}\left(\mathbf{A}_{C}\right)
\end{aligned}
$$

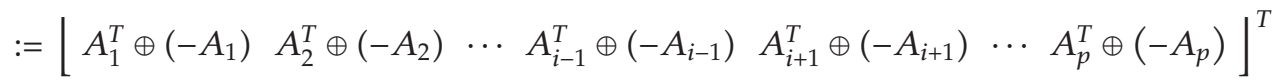

$$
\begin{aligned}
& \in \mathbf{R}^{(p-1) n^{2} \times n^{2}} \text {. }
\end{aligned}
$$

Then $A_{i} \in \mathbf{A}_{C} ; \forall i \in \bar{p}$ if and only if $v\left(A_{i}\right) \in \operatorname{Ker} N_{i}\left(\mathbf{A}_{C}\right) ; \forall i \in \bar{p}$. 
(iii) One has

$$
\begin{aligned}
M C_{\mathbf{A}_{C}} & :=\left\{X \in \mathbf{R}^{n \times n}: v(X) \in \bigcap_{i \in \bar{p}} \operatorname{Ker}\left(A_{i} \oplus\left(-A_{i}^{T}\right)\right) ; A_{i} \in \mathbf{A}_{C}\right\} \\
& =\left\{X \in \mathbf{R}^{n \times n}: v(X) \in \operatorname{Ker} N\left(\mathbf{A}_{C}\right)\right\} \supset C_{\mathbf{A}_{C}} \supset \mathbf{A}_{C} \supset\{0\} \in \mathbf{R}^{n \times n},
\end{aligned}
$$

where $N\left(\mathbf{A}_{C}\right):=\left[A_{1}^{T} \oplus\left(-A_{1}\right) \quad A_{2}^{T} \oplus\left(-A_{2}\right) \quad \cdots \quad A_{p}^{T} \oplus\left(-A_{p}\right)\right]^{T} \in \mathbf{R}^{p n^{2} \times n^{2}}, A_{i} \in \mathbf{A}_{C}$.

(iv) One has

$$
\begin{aligned}
\overline{M C_{\mathbf{A}_{C}}} & :=\left\{X \in \mathbf{R}^{n \times n}: v(X) \in \bigcup_{i \in \bar{p}} \operatorname{Im}\left(A_{i} \oplus\left(-A_{i}^{T}\right)\right) ; A_{i} \in \mathbf{A}_{C}\right\} \\
& =\left\{X \in \mathbf{R}^{n \times n}: v(X) \in \operatorname{Im} N\left(\mathbf{A}_{C}\right)\right\} .
\end{aligned}
$$

(v) One has

$$
\begin{aligned}
C_{\mathbf{A}} & :=\left\{X \in \mathbf{R}^{n \times n}: v(X) \in \bigcap_{i \in \bar{p}} \operatorname{Ker}\left(A_{i} \oplus\left(-A_{i}^{T}\right)\right) ; A_{i} \in \mathbf{A}\right\} \\
& =\left\{X \in \mathbf{R}^{n \times n}: v(X) \in \operatorname{Ker} N(\mathbf{A})\right\},
\end{aligned}
$$

where $N(\mathbf{A}):=\left[A_{1}^{T} \oplus\left(-A_{1}\right) \quad A_{2}^{T} \oplus\left(-A_{2}\right) \quad \cdots \quad A_{p}^{T} \oplus\left(-A_{p}\right)\right]^{T} \in \mathbf{R}^{p n^{2} \times n^{2}}, A_{i} \in \mathbf{A}$.

(vi) One has

$$
\begin{aligned}
\bar{C}_{\mathbf{A}} & :=\left\{X \in \mathbf{R}^{n \times n}: v(X) \in \bigcup_{i \in \bar{p}} \operatorname{Im}\left(A_{i} \oplus\left(-A_{i}^{T}\right)\right) ; A_{i} \in \mathbf{A}\right\} \\
& =\left\{X \in \mathbf{R}^{n \times n}: v(X) \in \operatorname{Im} N(\mathbf{A})\right\} .
\end{aligned}
$$

Concerning Proposition 3.1(v)-(vi), note that if $X \in \bar{C}_{\mathbf{A}}$, then $X \neq 0$ since $\mathbf{R}^{n \times n} \ni 0 \in C_{\mathbf{A}}$. The following result is related to the rank defectiveness of the matrix $N\left(\mathbf{A}_{C}\right)$ and any of their submatrices since $\mathbf{A}_{C}$ is a set of pair wise commuting matrices.

Proposition 3.2. The following properties hold:

$$
n^{2}>\operatorname{rank} N\left(\mathbf{A}_{C}\right) \geq \operatorname{rank} N_{i}\left(\mathbf{A}_{C}\right) \geq \operatorname{rank}\left(A_{j} \oplus\left(-A_{j}^{T}\right)\right) ; \quad \forall A_{j} \in \mathbf{A}_{C} ; \forall i, j \in \bar{p}
$$


and, equivalently,

$$
\begin{aligned}
\operatorname{det}\left(N^{T}\left(\mathbf{A}_{C}\right) N\left(\mathbf{A}_{C}\right)\right) & =\operatorname{det}\left(N_{i}^{T}\left(\mathbf{A}_{C}\right) N_{i}\left(\mathbf{A}_{C}\right)\right)=\operatorname{det}\left(A_{j} \oplus\left(-A_{j}^{T}\right)\right) \\
& =0 ; \quad \forall A_{j} \in \mathbf{A}_{C} ; \forall i, j \in \bar{n} .
\end{aligned}
$$

Results related to sufficient conditions for a set of matrices to pair wise commute are abundant in literature. For instance, diagonal matrices are always pair wise commuting. Any sets of matrices obtained via multiplication by real scalars with any given arbitrary matrix are sets of pair wise commuting matrices. Any set of matrices obtained by linear combinations of one of the above sets consists also of pair wise commuting matrices. Any matrix commutes with any of its matrix functions, and so forth. In the following, a simple, although restrictive, sufficient condition for rank defectiveness of $N(\mathbf{A})$ of some set $\mathbf{A}$ of $p$ square real $n$-matrices is discussed. Such a condition may be useful as a practical test to elucidate the existence of a nonzero $n$-square matrix which commutes with all matrices in this set. Another useful test obtained from the following result relies on a necessary condition to elucidate if the given set consists of pair wise commuting matrices.

Theorem 3.3. Consider any arbitrary set of nonzero $n$-square real matrices $\mathbf{A}:=\left\{A_{1}, A_{2}, \ldots, A_{p}\right\}$ for any integer $p \geq 1$ and define matrices:

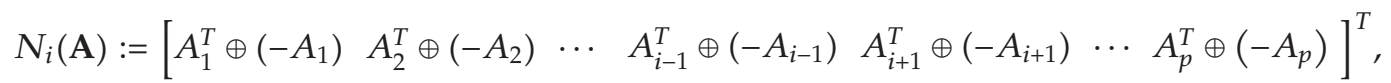

$$
\begin{aligned}
& N(\mathbf{A}):=\left\lfloor\begin{array}{llll}
A_{1}^{T} \oplus\left(-A_{1}\right) & A_{2}^{T} \oplus\left(-A_{2}\right) & \cdots & A_{p}^{T} \oplus\left(-A_{p}\right)
\end{array}\right\rfloor^{T} .
\end{aligned}
$$

Then, the following properties hold:

(i) $\operatorname{rank}\left(A_{i} \oplus\left(-A_{i}\right)\right) \leq \operatorname{rank} N_{i}(\mathbf{A}) \leq \operatorname{rank} N(\mathbf{A})<n^{2} ; \forall i \in \bar{p}$.

(ii) $\bigcap_{i \in \bar{p}} \operatorname{Ker}\left(A_{i} \oplus\left(-A_{i}^{T}\right)\right) \neq\{0\}$ so that

$$
\begin{gathered}
\exists X(\neq 0) \in C_{\mathbf{A}}, \quad X \in C_{\mathbf{A}} \Longleftrightarrow v(X) \in \bigcap_{i \in \bar{p}} \operatorname{Ker}\left(A_{i} \oplus\left(-A_{i}^{T}\right)\right), \\
X \in \bar{C}_{\mathbf{A}} \Longleftrightarrow v(X) \in \bigcup_{i \in \bar{p}} \operatorname{Im}\left(A_{i} \oplus\left(-A_{i}^{T}\right)\right) .
\end{gathered}
$$


(iii) If $\mathbf{A}=\mathbf{A}_{C}$ is a set of pair wise commuting matrices then

$$
\begin{aligned}
v\left(A_{i}\right) & \in \bigcap_{j \in \bar{p} \mid \bar{i}} \operatorname{Ker}\left(A_{j} \oplus\left(-A_{j}^{T}\right)\right) ; \quad \forall i \in \bar{p} \\
& \Longleftrightarrow v\left(A_{i}\right) \in \bigcap_{i \in \bar{p}} \operatorname{Ker}\left(A_{i} \oplus\left(-A_{i}^{T}\right)\right) ; \quad \forall i \in \bar{p} \\
& \Longleftrightarrow v\left(A_{i}\right) \in \bigcap_{i \in \bar{p} \backslash\{i\}} \operatorname{Ker}\left(A_{i} \oplus\left(-A_{i}^{T}\right)\right) ; \quad \forall i \in \bar{p} .
\end{aligned}
$$

(iv) One has

$$
M \mathbf{A}_{C}:=\left\{X \in \mathbf{R}^{n \times n}: v(X) \bigcap_{i \in \bar{p}} \operatorname{Ker}\left(A_{i} \oplus\left(-A_{i}^{T}\right)\right), \forall A_{i} \in \mathbf{A}_{C}\right\} \supset \mathbf{A}_{C} \cup\{0\} \in \mathbf{R}^{n \times n}
$$

with the above set inclusion being proper.

Note that Theorem 3.3(ii) extends Proposition 3.1(v) since it is proved that $C_{\mathrm{A}} \backslash\{0\} \neq \emptyset$ because all nonzero $\mathbf{R}^{n \times n} \ni \Lambda=\operatorname{diag}(\lambda \quad \lambda \cdots \lambda) \in C_{\mathbf{A}}$ for any $\mathbf{R} \ni \lambda \neq 0$ and any set of matrices A. Note that Theorem 3.3(iii) establishes that $v\left(A_{i}\right) \in \bigcap_{i \in \bar{p} \backslash\{i\}} \operatorname{Ker}\left(A_{j} \oplus\left(-A_{j}^{T}\right)\right) ; \forall i \in \bar{p}$ is a necessary and sufficient condition for the set to be a set of commuting matrices $\mathbf{A}$ being simpler to test (by taking advantage of the symmetry property of the commutators) than the equivalent condition $v\left(A_{i}\right) \in \bigcap_{i \in \bar{p}} \operatorname{Ker}\left(A_{j} \oplus\left(-A_{j}^{T}\right)\right) ; \forall i \in \bar{p}$. Further results about pair wise commuting matrices or the existence of nonzero commuting matrices with a given set are obtained in the subsequent result based on the Kronecker sum of relevant Jordan canonical forms.

Theorem 3.4. The following properties hold for any given set of $n$-square real matrices $\mathbf{A}=$ $\left\{A_{1}, A_{2}, \ldots, A_{p}\right\}$.

(i) The set $C_{\mathbf{A}}$ of matrices $X \in \mathbf{R}^{n \times n}$ which commute with all matrices in $\mathbf{A}$ is defined by:

$$
\begin{aligned}
C_{\mathbf{A}} & :=\left\{X \in \mathbf{R}^{n \times n}: v(X) \in \bigcap_{i=1}^{p}\left(\operatorname{Ker}\left[\left(J_{A_{i}} \oplus\left(-J_{A_{i}}^{T}\right)\right)\left(P_{i}^{-1} \otimes P_{i}^{-T}\right)\right]\right)\right\} \\
& =\left\{X \in \mathbf{R}^{n \times n}: v(X) \in \bigcap_{i=1}^{p}\left(\operatorname{Im}\left(\left(P_{i} \otimes P_{i}^{-1}\right)\left(Y_{i}\right)\right)\right) \wedge Y_{i} \in \operatorname{Ker}\left(J_{A_{i}} \oplus\left(-J_{A_{i}}^{T}\right)\right) ; \forall i \in \bar{p}\right\} \\
& =\left\{X \in \mathbf{R}^{n \times n}: v(X) \in \bigcap_{i=1}^{p}\left(\operatorname{Im}\left(\left(P_{i} \otimes P_{i}^{-1}\right)(Y)\right)\right), Y \in \bigcap_{i=1}^{p}\left(\operatorname{Ker}\left(J_{A_{i}} \oplus\left(-J_{A_{i}}^{T}\right)\right)\right)\right\}
\end{aligned}
$$

where $P_{i} \in \mathbf{R}^{n \times n}$ is a nonsingular transformation matrix such that $A_{i} \sim J_{A_{i}}=P_{i}^{-1} A_{i} P_{i}, J_{A_{i}}$ being the Jordan canonical form of $A_{i}$. 
(ii) One has

$$
\begin{aligned}
\operatorname{dim} \operatorname{span}\left\{v(X): X \in C_{\mathbf{A}}\right\} & \leq \min _{i \in \bar{p}} \operatorname{dim}\left(\operatorname{Ker}\left(J_{A_{i}} \oplus\left(-J_{A_{i}}^{T}\right)\right)\right) \\
& =\min _{i \in \bar{p}} \bar{v}_{i}(0)=\min _{i \in \bar{p}}\left(\sum_{j=1}^{\rho_{i}} v_{i j}^{2}\right) \leq \min _{i \in \bar{p}}\left(\sum_{i=1}^{\rho_{i}} \mu_{i j}^{2}\right) \leq \min _{i \in \bar{p}}\left(\bar{\mu}_{i}(0)\right),
\end{aligned}
$$

where $\bar{v}_{i}(0)$ and $v_{i j}$ are, respectively, the geometric multiplicities of $0 \in \sigma\left(A_{i} \oplus\left(-A_{i}^{T}\right)\right)$ and $\lambda_{i j} \in \sigma\left(A_{i}\right)$ and $\bar{\mu}_{i}(0)$ and $\mu_{i j}$ are, respectively, the algebraic multiplicities of $0 \in \sigma\left(A_{i} \oplus\left(-A_{i}^{T}\right)\right)$ and $\lambda_{i j} \in \sigma\left(A_{i}\right)$; $\forall j \in \rho_{i}$ (the number of distinct eigenvalues of $A_{i}$ ), $\forall i \in \bar{p}$.

(iii) The set $\mathbf{A}$ consists of pair wise commuting matrices, namely $C_{\mathbf{A}}=M C_{\mathbf{A}}$, if and only if $v\left(A_{j}\right) \in \bigcap_{i(\neq j)=1}^{p}\left(\operatorname{Ker}\left[\left(J_{A_{i}} \oplus\left(-J_{A_{i}}^{T}\right)\right)\left(P_{i}^{-1} \otimes P_{i}^{-T}\right)\right]\right) ; \forall j \in \bar{p}$. Equivalent conditions follow from the second and third equivalent definitions of $C_{\mathbf{A}}$ in Property (i).

Theorems 3.3 and 3.4 are concerned with $\mathrm{MC}_{\mathbf{A}} \neq\{0\} \in \mathbf{R}^{n \times n}$ for an arbitrary set of real square matrices $\mathrm{A}$ and for a pair wise-commuting set, respectively.

\section{Further Results and Extensions}

The extensions of the results for commutation of complex matrices are direct in several ways. It is first possible to decompose the commutator in its real and imaginary part and then apply the results of Sections 2 and 3 for real matrices to both parts as follows. Let $A=A_{\mathrm{re}}+\mathbf{i} A_{\mathrm{im}}$ and $B=B_{\text {re }}+\mathbf{i} B_{\text {im }}$ be complex matrices in $\mathbf{C}^{n \times n}$ with $A_{\text {re }}$ and $B_{\text {re }}$ being their respective real parts, and $A_{\mathrm{im}}$ and $B_{\mathrm{im}}$, all in $\mathbf{R}^{n \times n}$, their respective imaginary parts, and $\mathbf{i}=\sqrt{-1}$ is the imaginary complex unity. Direct computations with the commutator of $A$ and $B$ yield

$$
[A, B]=\left(\left[A_{\mathrm{re}}, B_{\mathrm{re}}\right]-\left[A_{\mathrm{im}}, B_{\mathrm{im}}\right]\right)+\mathbf{i}\left(\left[A_{\mathrm{im}}, B_{\mathrm{re}}\right]+\left[A_{\mathrm{re}}, B_{\mathrm{im}}\right]\right) .
$$

The following three results are direct and allow to reduce the problem of commutation of a pair of complex matrices to the discussion of four real commutators.

Proposition 4.1. One has $B \in C_{A} \Leftrightarrow\left(\left(\left[A_{r e}, B_{r e}\right]=\left[A_{i m}, B_{i m}\right]\right) \wedge\left(\left[A_{i m}, B_{r e}\right]=\left[B_{i m}, A_{r e}\right]\right)\right)$.

Proposition 4.2. One has $\left(B_{r e} \in\left(C_{A_{r e}} \cap C_{A_{i m}}\right) \wedge B_{i m} \in\left(C_{A_{i m}} \cap C_{A_{r e}}\right)\right) \Rightarrow B \in C_{A}$.

Proposition 4.3. One has $\left(A_{r e} \in\left(C_{B_{r e}} \cap C_{B_{i m}}\right) \wedge A_{i m} \in\left(C_{B_{i m}} \cap C_{B_{r e}}\right)\right) \Rightarrow B \in C_{A}$.

Proposition 4.1 yields to the subsequent result.

Theorem 4.4. The following properties hold.

(i) Assume that the matrices $A$ and $B_{r e}$ are given. Then, $B \in C_{A}$ if and only if $B_{\text {im }}$ satisfies the following linear algebraic equation:

$$
\left[\begin{array}{c}
A_{r e} \oplus\left(-A_{r e}^{T}\right) \\
A_{i m} \oplus\left(-A_{i m}^{T}\right)
\end{array}\right] v\left(B_{r e}\right)=\left[\begin{array}{c}
A_{i m} \oplus\left(-A_{i m}^{T}\right) \\
A_{r e} \oplus\left(-A_{r e}^{T}\right)
\end{array}\right] v\left(B_{i m}\right)
$$


for which a necessary condition is

$$
\left.\operatorname{rank}\left[\begin{array}{c}
A_{i m} \oplus\left(-A_{i m}^{T}\right) \\
A_{r e} \oplus\left(-A_{r e}^{T}\right)
\end{array}\right]=\operatorname{rank}\left[\begin{array}{c}
A_{i m} \oplus\left(-A_{i m}^{T}\right) \\
A_{r e} \oplus\left(-A_{r e}^{T}\right)
\end{array} \quad \begin{array}{c}
A_{r e} \oplus\left(-A_{r e}^{T}\right) \\
A_{i m} \oplus\left(-A_{i m}^{T}\right)
\end{array}\right) v\left(B_{r e}\right)\right]
$$

(ii) Assume that the matrices $A$ and $B_{i m e}$ are given. Then, $B \in C_{A}$ if and only if $B_{r e}$ satisfies (4.2) for which a necessary condition is

$$
\operatorname{rank}\left[\begin{array}{c}
A_{r e} \oplus\left(-A_{r e}^{T}\right) \\
A_{i m} \oplus\left(-A_{i m}^{T}\right)
\end{array}\right]=\operatorname{rank}\left[\begin{array}{c}
A_{r e} \oplus\left(-A_{r e}^{T}\right) \\
A_{i m} \oplus\left(-A_{i m}^{T}\right)
\end{array} \quad\left(\begin{array}{c}
A_{i m} \oplus\left(-A_{i m}^{T}\right) \\
A_{r e} \oplus\left(-A_{r e}^{T}\right)
\end{array}\right) v\left(B_{i m}\right)\right] .
$$

(iii) Also, $\exists B \neq 0$ such that $B \in C_{A}$ with $B_{r e}=0$ and $\exists B \neq 0$ such that $B \in C_{A}$ with $B_{\text {im }}=0$.

A more general result than Theorem 4.4 is the following.

Theorem 4.5. The following properties hold.

(i) $B \in C_{A} \cap C^{n \times n}$ if and only if $v(B)$ is a solution to the following linear algebraic system:

$$
\left[\begin{array}{lr}
A_{r e} \oplus\left(-A_{r e}^{T}\right) & \left(-A_{i m}\right) \oplus\left(A_{i m}^{T}\right) \\
A_{i m} \oplus\left(-A_{i m}^{T}\right) & \left(-A_{r e}\right) \oplus\left(A_{r e}^{T}\right)
\end{array}\right]\left[\begin{array}{l}
v\left(B_{r e}\right) \\
v\left(B_{i m}\right)
\end{array}\right]=0 .
$$

Nonzero solutions $B \in C_{A}$, satisfying

$$
\left[\begin{array}{c}
v\left(B_{r e}\right) \\
v\left(B_{i m}\right)
\end{array}\right] \in \operatorname{Ker}\left[\begin{array}{ll}
A_{r e} \oplus\left(-A_{r e}^{T}\right) & \left(-A_{i m}\right) \oplus\left(A_{i m}^{T}\right) \\
A_{i m} \oplus\left(-A_{i m}^{T}\right) & \left(-A_{r e}\right) \oplus\left(A_{r e}^{T}\right)
\end{array}\right]
$$

always exist since

$$
\operatorname{Ker}\left[\begin{array}{ll}
A_{r e} \oplus\left(-A_{r e}^{T}\right) & \left(-A_{i m}\right) \oplus\left(A_{i m}^{T}\right) \\
A_{i m} \oplus\left(-A_{i m}^{T}\right) & \left(-A_{r e}\right) \oplus\left(A_{r e}^{T}\right)
\end{array}\right] \neq\{0\} \in \mathbf{R}^{2 n^{2}},
$$

and equivalently, since

$$
\operatorname{rank}\left[\begin{array}{lr}
A_{r e} \oplus\left(-A_{r e}^{T}\right) & \left(-A_{i m}\right) \oplus\left(A_{i m}^{T}\right) \\
A_{i m} \oplus\left(-A_{i m}^{T}\right) & \left(-A_{r e}\right) \oplus\left(A_{r e}^{T}\right)
\end{array}\right]<2 n^{2} .
$$

(ii) Property (ii) is equivalent to

$$
B \in C_{A} \Longleftrightarrow\left(A \oplus\left(-A^{*}\right)\right) v(B)=0
$$

which has always nonzero solutions since $\operatorname{rank}\left(A \oplus\left(-A^{*}\right)\right)<n^{2}$. 
The various results of Section 3 for a set of distinct complex matrices to pair wise commute and for characterizing the set of complex matrices which commute with those in a given set may be discussed by more general algebraic systems like the above one with four block matrices

$$
\left[\begin{array}{ll}
A_{j \mathrm{re}} \oplus\left(-A_{2 \mathrm{re}}^{T}\right) & \left(-A_{j \mathrm{im}}\right) \oplus\left(A_{j \mathrm{im}}^{T}\right) \\
A_{j \mathrm{im}} \oplus\left(-A_{2 \mathrm{im}}^{T}\right) & \left(-A j_{2 \mathrm{re}}\right) \oplus\left(A_{j \mathrm{re}}^{T}\right)
\end{array}\right]
$$

for each $j \in \bar{p}$ in the whole algebraic system. Theorem 4.5 extends directly for sets of complex matrices commuting with a given one and complex matrices commuting with a set of commuting complex matrices as follows.

Theorem 4.6. The following properties hold.

(i) Consider the sets of nonzero distinct complex matrices $\mathbf{A}:=\left\{A_{i} \in \mathbf{C}^{n \times n}: i \in \bar{p}\right\}$ and $C_{\mathbf{A}}:=\left\{X \in \mathbf{C}^{n \times n}:\left[X, A_{i}\right]=0 ; A_{i} \in \mathbf{A}, \forall i \in \bar{p}\right\}$ for $p \geq 2$. Thus, $C_{\mathbf{A}} \ni X=X_{r e}+\mathbf{i} X_{r e}$ if and only if

$$
\left[\begin{array}{cc}
A_{1 r e} \oplus\left(-A_{1 r e}^{T}\right) & \left(-A_{1 i m}\right) \oplus\left(A_{1 i m}^{T}\right) \\
A_{1 i m} \oplus\left(-A_{1 i m}^{T}\right) & \left(-A_{1 r e}\right) \oplus\left(A_{1 r e}^{T}\right) \\
A_{2 r e} \oplus\left(-A_{2 r e}^{T}\right) & \left(-A_{2 i m}\right) \oplus\left(A_{2 i m}^{T}\right) \\
A_{2 i m} \oplus\left(-A_{2 i m}^{T}\right) & \left(-A_{2 r e}\right) \oplus\left(A_{2 r e}^{T}\right) \\
\vdots \\
A_{\text {pre }} \oplus\left(-A_{\text {pre }}^{T}\right) & \left(-A_{\text {pim }}\right) \oplus\left(A_{\text {pim }}^{T}\right) \\
A_{\text {pim }} \oplus\left(-A_{\text {pim }}^{T}\right) & \left(-A_{\text {pre }}\right) \oplus\left(A_{\text {pre }}^{T}\right)
\end{array}\right]\left[\begin{array}{c}
v\left(X_{\text {re }}\right) \\
v\left(X_{\text {im }}\right)
\end{array}\right]=0,
$$

and a nonzero solution $X \in C_{\mathrm{A}}$ exists since the rank of the coefficient matrix of (4.11) is less than $2 n^{2}$.

(ii) Consider the sets of nonzero distinct commuting complex matrices $\mathbf{A}_{C}:=\left\{A_{i} \in \mathbf{C}^{n \times n}: i \in\right.$ $\bar{p}\}$ and $M C_{\mathbf{A}}:=\left\{X \in \mathbf{C}^{n \times n}:\left[X, A_{i}\right]=0 ; A_{i} \in \mathbf{A}, \forall i \in \bar{p}\right\}$ for $p \geq 2$. Thus, $M C_{\mathbf{A}} \ni X=X_{r e}+\mathbf{i} X_{r e}$ if and only if $v\left(X_{r e}\right)$ and $v\left(X_{i m}\right)$ are solutions to (4.11). equations:

(iii) Properties (i) and (ii) are equivalently formulated by from the algebraic set of complex

$$
\left[\begin{array}{llll}
A_{1}^{*} \oplus\left(-A_{1}\right) & A_{2}^{*} \oplus\left(-A_{2}\right) & \cdots & A_{p}^{*} \oplus\left(-A_{p}\right)
\end{array}\right]^{*} v(X)=0 .
$$

Remark 4.7. Note that all the proved results of Sections 2 and 3 are directly extendable for complex commuting matrices, by simple replacements of transposes by conjugate transposes, without requiring a separate decomposition in real and imaginary parts as discussed in Theorems 4.5(ii) and 4.6(iii).

Let $f: \mathbf{C} \rightarrow \mathbf{C}$ be an analytic function in an open set $D \supset \sigma(A)$ for some matrix $A \in \mathbf{C}^{n \times n}$ and let $p(\lambda)$ be a polynomial fulfilling $p^{(i)}\left(\lambda_{k}\right)=f^{(i)}\left(\lambda_{k}\right) ; \forall k \in \sigma(A), \forall i \in \overline{m_{k}-1} \cup$ $\{0\} ; \forall k \in \mu$ (the number of distinct elements in $\sigma(A)$ ), where $m_{k}$ is the index of $\lambda_{k}$, that is, 
its multiplicity in the minimal polynomial of $A$. Then, $f(A)$ is a function of a matrix $A$ if $f(A)=p(A)$, [8]. Some results follow concerning the commutators of functions of matrices.

Theorem 4.8. Consider a nonzero matrix $B \in C_{A} \cap \mathrm{C}^{n \times n}$ for any given nonzero $A \in \mathrm{C}^{n \times n}$. Then, $f(B) \in C_{A} \cap C^{n \times n}$, and equivalently $v(f(B)) \in \operatorname{Ker}\left(A \oplus\left(-A^{*}\right)\right)$, for any function $f: \mathbf{C}^{n \times n} \rightarrow \mathbf{C}^{n \times n}$ of the matrix $B$.

The following corollaries are direct from Theorem 4.8 from the subsequent facts:

(1) $A \in C_{A} ; \forall A \in \mathbf{C}^{n \times n}$,

(2) One has

$$
\begin{aligned}
{[A, B] } & =0 \Longrightarrow[A, g(B)]=0 \Longrightarrow[f(A), g(B)]=[p(A), g(B)]=\sum_{i=0}^{\mu} \alpha_{i}\left[A^{i}, g(B)\right] \\
& =\sum_{i=0}^{\mu} \alpha_{i} A^{i-1}[A, g(B)]=0 \Longleftrightarrow g(B) \in C_{f(A)} \cap C^{n \times n},
\end{aligned}
$$

where $f(A)=p(A)$, from the definition of $f$ being a function of the matrix $A$, with $p(\lambda)$ being a polynomial fulfilling $p^{(i)}\left(\lambda_{k}\right)=f^{(i)}\left(\lambda_{k}\right) ; \forall k \in \sigma(A), \forall i \in \overline{m_{k}-1} \cup\{0\}$; $\forall k \in \mu$ (the number of distinct elements in $\sigma(A)$ ), where $m_{k}$ is the index of $\lambda_{k}$, that is, its multiplicity in the minimal polynomial of $A$.

(3) Theorem 4.8 is extendable for any countable set $\left\{f_{i}(B)\right\}$ of matrix functions of $B$.

Corollary 4.9. Consider a nonzero matrix $B \in C_{A} \cap \mathbf{C}^{n \times n}$ for any given nonzero $A \in \mathbf{C}^{n \times n}$. Then, $g(B) \in C_{f(A)} \cap C^{n \times n}$ for any function $f: \mathbf{C}^{n \times n} \rightarrow \mathbf{C}^{n \times n}$ of the matrix $A$ and any function $g: \mathbf{C}^{n \times n} \rightarrow$ $\mathrm{C}^{n \times n}$ of the matrix $B$.

Corollary 4.10. $f(A) \in C_{A} \cap C^{n \times n}$, and equivalently $v(f(A)) \in \operatorname{Ker}\left(A \oplus\left(-A^{*}\right)\right)$, for any function $f: \mathbf{C}^{n \times n} \rightarrow \mathbf{C}^{n \times n}$ of the matrix $A$.

Corollary 4.11. If $B \in C_{A} \cap C^{n \times n}$ then any countable set of function matrices $\left\{f_{i}(B)\right\}$ is $C_{A}$ and in $M C_{A}$.

Corollary 4.12. Consider any countable set of function matrices $C_{F}:=\left\{f_{i}(A) ; \forall i \in \bar{p}\right\} \subset C_{A}$ for any given nonzero $A \in \mathbf{C}^{n \times n}$. Then, $\bigcap_{f_{i} \in C_{F}}\left(\operatorname{Ker}\left(f_{i}(A) \oplus\left(-f_{i}\left(A^{*}\right)\right)\right)\right) \supset \operatorname{Ker}\left(A \oplus\left(-A^{*}\right)\right)$.

Note that matrices which commute and are simultaneously triangularizable through the same similarity transformation maintain a zero commutator after such a transformation is performed.

Theorem 4.13. Assume that $B \in C_{A} \cap C^{n \times n}$. Thus, $\Lambda_{B} \in C_{\Lambda_{A}} \cap C^{n \times n}$ provided that there exists a nonsingular matrix $T \in \mathrm{C}^{n \times n}$ such that $\Lambda_{A}=T^{-1} A T$ and $\Lambda_{B}=T^{-1} B T$.

A direct consequence of Theorem 4.13 is that if a set of matrices are simultaneously triangularizable to their real canonical forms by a common transformation matrix then the pair wise commuting properties are identical to those of their respective Jordan forms. 


\section{Appendices}

\section{A. Proofs of the Results of Section 2}

Proof of Proposition 2.1. (i)-(ii) First note by inspection that $\emptyset \neq C_{A} \supset\{0, A\} ; \forall A \in \mathbf{R}^{n \times n}$. Also,

$$
\begin{aligned}
{[A, X] } & =A X-X A=\left(A \otimes I_{n}-I_{n} \otimes A^{T}\right) v(X) \\
& =\left(A \oplus\left(-A^{T}\right)\right) v(X)=0 \Longrightarrow v(X) \in \operatorname{Ker}\left(A \oplus\left(-A^{T}\right)\right)
\end{aligned}
$$

and Proposition 2.1(i)-(ii) has been proved since there is an isomorphism $f: \mathbf{R}^{n^{2}} \leftrightarrow \mathbf{R}^{n \times n}$ defined by $f(v(X))=X ; \forall X \in \mathbf{R}^{n \times n}$ for $v(X)=\left(x_{1}^{T}, x_{2}^{T}, \ldots, x_{n}^{T}\right)^{T} \in \mathbf{R}^{n^{2}}$ if $x_{i}^{T}:=\left(x_{i 1}, x_{i 2}, \ldots, x_{i n}\right)$ is the $i$ th row of the square matrix $X$.

(iii) It is a direct consequence of Proposition 2.1(iii) and the symmetry property of the commutator of two commuting matrices $B \in C_{A} \Leftrightarrow[A, B]=[B, A]=0 \Leftrightarrow A \in C_{B}$.

Proof of Proposition 2.2. $[A, A]=0 ; \forall A \in \mathbf{R}^{n \times n} \Rightarrow \exists \mathbf{R}^{n^{2}} \ni 0 \neq v(A) \in \operatorname{Ker}\left(A \oplus\left(-A^{T}\right)\right) ; \forall A \in$ $\mathbf{R}^{n \times n}$. As a result,

$$
\operatorname{Ker}\left(A \oplus\left(-A^{T}\right)\right) \neq 0 \in \mathbf{R}^{n^{2}} ; \quad \forall A \in \mathbf{R}^{n \times n} \Longleftrightarrow \operatorname{rank}\left(A \oplus\left(-A^{T}\right)\right)<n^{2} ; \quad \forall A \in \mathbf{R}^{n \times n}
$$

so that $0 \in \sigma\left(A \oplus\left(-A^{T}\right)\right)$.

Also, $\exists X(\neq 0) \in \mathbf{R}^{n \times n}:[A, X]=0 \Leftrightarrow X \in C_{A}$ since $\operatorname{Ker}\left(A \oplus\left(-A^{T}\right)\right) \neq 0 \in \mathbf{R}^{n^{2}}$.

Proposition 2.2 has been proved.

Proof of Theorem 2.3. (i) Note that

$$
\begin{aligned}
\sigma(A) & =\sigma\left(A^{T}\right) \Longrightarrow \sigma\left(A \oplus\left(-A^{T}\right)\right):=\left\{\mathbf{C} \ni \eta=\lambda_{k}-\lambda_{\ell} ; \forall \lambda_{k}, \lambda_{\ell} \in \sigma(A) ; \forall k, \ell \in \bar{n}\right\} \\
& =\sigma_{0}\left(A \oplus\left(-A^{T}\right)\right) \cup \overline{\sigma_{0}\left(A \oplus\left(-A^{T}\right)\right)}
\end{aligned}
$$

where

$$
\begin{aligned}
\sigma_{0}\left(A \oplus\left(-A^{T}\right)\right) & =\left\{\lambda \in \sigma\left(A \oplus\left(-A^{T}\right)\right): \lambda=0\right\}, \\
\overline{\sigma_{0}\left(A \oplus\left(-A^{T}\right)\right)} & =\left\{\lambda \in \sigma\left(A \oplus\left(-A^{T}\right)\right): \lambda \neq 0\right\}=\sigma\left(A \oplus\left(-A^{T}\right)\right) \backslash \sigma_{0}\left(A \oplus\left(-A^{T}\right)\right) .
\end{aligned}
$$

Furthermore, $\sigma\left(A \oplus\left(-A^{T}\right)\right):=\left\{\mathbf{C} \ni \lambda=\lambda_{j}-\lambda_{i}: \lambda_{i}, \lambda_{j} \in \sigma(A) ; \forall i, j \in \bar{n}\right\}$ and $z_{i} \otimes x_{j}$ is a right eigenvector of $A \oplus\left(-A^{T}\right)$ associated with its eigenvalue $\bar{\lambda}_{j i}=\lambda_{j}-\lambda_{i} \cdot \lambda=\lambda_{j}-\lambda_{i} \in \sigma\left(A \oplus\left(-A^{T}\right)\right)$ has algebraic and geometric multiplicities $\bar{\mu}_{j i}$ and $\bar{v}_{j i}$, respectively; $\forall i, j \in \bar{n}$, since $x_{j}$ and $z_{i}$ are, respectively, the right eigenvectors of $A$ and $A^{T}$ with associated eigenvalues $\lambda_{j}$ and $\lambda_{i} ; \forall i, j \in \bar{n}$.

Let $J_{A}$ be the Jordan canonical form of $A$. It is first proved that there exists a nonsingular $T \in \mathbf{R}^{n^{2} \times n^{2}}$ such that $J_{A} \oplus\left(-J_{A^{T}}\right)=T^{-1}\left(A \oplus\left(-A^{T}\right)\right) T$. The proof is made by 
direct verification by using the properties of the Kronecker product, with $T=P \otimes P^{T}$ for a nonsingular $P \in \mathbf{R}^{n \times n}$ such that $A \sim J_{A}=P^{-1} A P$, as follows:

$$
\begin{aligned}
T^{-1}\left(A \oplus\left(-A^{T}\right)\right) T & =\left(P \otimes P^{T}\right)^{-1}\left(A \otimes I_{n}\right)\left(P \otimes P^{T}\right)-\left(P \otimes P^{T}\right)^{-1}\left(I_{n} \otimes A^{T}\right)\left(P \otimes P^{T}\right) \\
& =\left(P^{-1} A P\right) \otimes\left(P^{-T} I_{n} P^{T}\right)-\left(P^{-1} I_{n} P\right) \otimes\left(P^{-T} A^{T} P^{T}\right) \\
& =\left(P^{-1} A P\right) \otimes I_{n}-I_{n} \otimes\left(P^{-T} A^{T} P^{T}\right) \\
& =J_{A} \otimes I_{n}-I_{n} \otimes J_{A^{T}}=J_{A} \otimes I_{n}+I_{n} \otimes\left(-J_{A^{T}}\right)=J_{A} \oplus\left(-J_{A^{T}}\right)
\end{aligned}
$$

and the result has been proved. Thus, $\operatorname{rank}\left(A \oplus\left(-A^{T}\right)\right)=\operatorname{rank}\left(J_{A} \oplus\left(-J_{A^{T}}\right)\right)$. It turns out that $P$ is, furthermore, unique except for multiplication by any nonzero real constant. Otherwise, if $T \neq P \otimes P^{T}$, then there would exist a nonsingular $Q \in \mathbf{R}^{n \times n}$ with $Q \neq \alpha I_{n} ; \forall \alpha \in \mathbf{R}$ such that $T=Q\left(P \otimes P^{T}\right)^{-1} Q$ so that $T^{-1}\left(A \oplus\left(-A^{T}\right)\right) T \neq J_{A} \oplus\left(-J_{A^{T}}\right)$ provided that

$$
\left(P \otimes P^{T}\right)^{-1}\left(A \oplus\left(-A^{T}\right)\right)\left(P \otimes P^{T}\right)=J_{A} \oplus\left(-J_{A^{T}}\right) .
$$

Thus, note that

$$
\begin{aligned}
\operatorname{card} \sigma\left(A \oplus\left(-A^{T}\right)\right) & =n^{2}=\sum_{i=1}^{\mu} \bar{\mu}_{i i}=\left(\sum_{i=1}^{\mu} \mu_{i}\right)^{2} \geq \bar{\mu}(0)=\sum_{i=1}^{\mu} \bar{\mu}_{i i}=\sum_{i=1}^{\mu} \mu_{i}^{2} \geq \bar{v} \\
& \geq \bar{v}(0)=\sum_{i=1}^{\mu} \bar{v}_{i i}=\sum_{i=1}^{\mu} v_{i}^{2}=\left(\sum_{i=1}^{\mu} \sum_{j=1}^{\mu} \bar{v}_{i j}\right)^{2}-2 \sum_{i=1}^{\mu} \sum_{j(\neq i)=1}^{\mu} \bar{v}_{i j} \\
& =\bar{v}-2 \sum_{i=1}^{\mu} \sum_{j(\neq i)=1}^{\mu} \bar{v}_{i j} \geq n .
\end{aligned}
$$

Those results follow directly from the properties of the Kronecker sum $A \oplus B$ of $n$-square real matrices $A$ and $B=-A^{T}$ since direct inspection leads to the following.

(1) $0 \in \sigma\left(A \oplus\left(-A^{T}\right)\right)$ with algebraic multiplicity $\bar{\mu}(0) \geq \sum_{i=1}^{\mu} \bar{\mu}_{i i}=\sum_{i=1}^{\mu} \mu_{i}^{2} \geq \sum_{i=1}^{\mu} v_{i}^{2} \geq n$ since there are at least $\sum_{i=1}^{n} \mu_{i}^{2}$ zeros in $\sigma\left(A \oplus\left(-A^{T}\right)\right)$ (i.e., the algebraic multiplicity of $0 \in \sigma\left(A \oplus\left(-A^{T}\right)\right)$ is at least $\left.\sum_{i=1}^{n} \mu_{i}^{2}\right)$ and since $v_{i} \geq 1 ; \forall i \in \bar{n}$. Also, a simple computation of the number of eigenvalues of $A \oplus\left(-A^{T}\right)$ yields card $\sigma\left(A \oplus\left(-A^{T}\right)\right)=$ $n^{2}=\sum_{i=1}^{\mu} \bar{\mu}_{i i}=\left(\sum_{i=1}^{\mu} \mu_{i}\right)^{2}$.

(2) The number of linearly independent vectors in $S$ is $\bar{v}=\sum_{i=1}^{\mu} \sum_{j=1}^{\mu} \bar{v}_{i j}=\left(\sum_{i=1}^{\mu} v_{i}\right)^{2} \geq$ $\sum_{i=1}^{\mu} \bar{v}_{i i}=\sum_{i=1}^{\mu} v_{i}^{2}$ since the total number of Jordan blocks in the Jordan canonical form of $A$ is $\sum_{i=1}^{\mu} v_{i}$. 
(3) The number of Jordan blocks associated with $0 \in \sigma\left(A \oplus\left(-A^{T}\right)\right)$ in the Jordan canonical form of $\left(A \oplus\left(-A^{T}\right)\right)$ is $\bar{v}(0)=\sum_{i=1}^{\mu} v_{i}^{2} \leq \bar{v}$, with $\bar{v}_{i i}=v_{i i}^{2} ; \forall i \in \bar{n}$. Thus,

$$
\begin{gathered}
\operatorname{card} \sigma_{0}\left(A \oplus\left(-A^{T}\right)\right)=\sum_{i=1}^{\mu} \bar{\mu}_{i i}=\sum_{i=1}^{\mu} \mu_{i}^{2}, \quad \operatorname{card} \bar{\sigma}_{0}\left(A \oplus\left(-A^{T}\right)\right)=n^{2}-\sum_{i=1}^{\mu} \mu_{i}^{2} \\
\operatorname{rank}\left(A \oplus\left(-A^{T}\right)\right)=n^{2}-\bar{v}(0)=n^{2}-\sum_{i=1}^{\mu} v_{i}^{2}, \quad \operatorname{dim} \operatorname{Ker}\left(A \oplus\left(-A^{T}\right)\right)=\bar{v}(0)=\sum_{i=1}^{\mu} v_{i}^{2}
\end{gathered}
$$

(4) There are at least $\bar{\nu}(0)$ linearly independent vectors in $S:=\operatorname{span}\left\{z_{i} \otimes x_{j}, \forall i, j \in \bar{n}\right\}$. Also, the total number of Jordan blocks in the Jordan canonical form of $\left(A \oplus\left(-A^{T}\right)\right)$ is $\bar{v}=\operatorname{dim} S=\left(\sum_{i=1}^{\mu} \sum_{j=1}^{\mu} \bar{v}_{i j}\right)=\left(\sum_{i=1}^{\mu} v_{i}\right)^{2}=\bar{v}(0)+2 \sum_{i=1}^{\mu} \sum_{j(\neq i)=1}^{\mu} \bar{v}_{i j} \geq \bar{v}(0)$.

Property (i) has been proved. Property (ii) follows directly from the orthogonality in $\mathbf{R}^{n^{2}}$ of its range and null subspaces.

Proof of Theorem 2.4. First note from Proposition 2.1 that $X \in C_{A}$ if and only if $(A \oplus$ $\left.\left(-A^{T}\right)\right) v(X)=0$ since $v(X) \in \operatorname{Ker}\left(A \oplus\left(-A^{T}\right)\right)$. Note also from Proposition 2.1 that $X \in \bar{C}_{A}$ if and only if $v(X) \in \operatorname{Im}\left(A \oplus\left(-A^{T}\right)\right)$. Thus, $X \in \bar{C}_{A}$ if and only if $v(X)$ is a solution to the algebraic compatible linear system:

$$
\left(A \oplus\left(-A^{T}\right)\right) v(X)=v(M)
$$

for any $M(\neq 0) \in \mathbf{R}^{n \times n}$ such that

$$
\operatorname{rank}\left(A \oplus\left(-A^{T}\right)\right)=\operatorname{rank}\left(A \oplus\left(-A^{T}\right), v(M)\right)=n^{2}-\bar{v}(0) .
$$

From Theorem 2.3, the nullity and the rank of $A \oplus\left(-A^{T}\right)$ are, respectively, $\operatorname{dim} \operatorname{Ker}(A \oplus$ $\left.\left(-A^{T}\right)\right)=\bar{v}(0) \quad \operatorname{rank}\left(A \oplus\left(-A^{T}\right)\right)=n^{2}-\bar{v}(0)$. Therefore, there exist permutation matrices $E, F \in \mathbf{R}^{n^{2} \times n^{2}}$ such that there exists an equivalence transformation:

$$
A \oplus\left(-A^{T}\right) \approx \bar{A}:=E\left(A \oplus\left(-A^{T}\right)\right) F=\operatorname{Block} \operatorname{matrix}\left(\bar{A}_{i j} ; i, j \in \overline{2}\right)
$$

such that $\bar{A}_{11}$ is square nonsingular and of order $\overline{\mathcal{v}}_{0}$. Define $\bar{M}=E M F \approx M(\neq 0) \in \mathbf{R}^{n \times n}$. Then, the linear algebraic systems $\left(A \oplus\left(-A^{T}\right)\right) v(X)=v(M)$, and

$$
\begin{aligned}
E\left(A \oplus\left(-A^{T}\right)\right) F v(\bar{X}) & =\left[\begin{array}{ll}
\bar{A}_{11} & \bar{A}_{12} \\
\bar{A}_{21} & \bar{A}_{22}
\end{array}\right]\left[\begin{array}{l}
v\left(\bar{X}_{1}\right) \\
v\left(\bar{X}_{2}\right)
\end{array}\right]=\left[\begin{array}{l}
v\left(\bar{M}_{1}\right) \\
v\left(\bar{M}_{2}\right)
\end{array}\right], \\
V\left(\bar{X}_{1}\right) & =\bar{A}_{11}^{-1}\left(v\left(\bar{M}_{1}\right)-\bar{A}_{12} v\left(\bar{X}_{2}\right)\right) \\
& \Longleftrightarrow\left(\bar{A}_{22}-\bar{A}_{21} \bar{A}_{11}^{-1} \bar{A}_{12}\right) V\left(\bar{X}_{2}\right)=v\left(\bar{M}_{2}\right)-\bar{A}_{21} \bar{A}_{11}^{-1} v\left(\bar{M}_{1}\right)
\end{aligned}
$$


are identical if $\bar{X}$ and $\bar{M}$ are defined according to $v(X)=F v(\bar{X})$ and $v(\bar{M})=E v(M)$. As a result, Properties (i) and (ii) follow directly from (A.12) for $M=\bar{M}=0$ and for any $M$ satisfying $\operatorname{rank}\left(A \oplus\left(-A^{T}\right)\right)=\operatorname{rank}\left(A \oplus\left(-A^{T}\right), v(M)\right)=n^{2}-\bar{v}(0)$, respectively.

\section{B. Proofs of the Results of Section 3}

Proof of Proposition 3.1. (i) The first part of Property (i) follows directly from Proposition 2.1 since all the matrices of $\mathbf{A}_{C}$ pair wise commute and any arbitrary matrix commutes with itself (thus $j=i$ may be removed from the intersections of kernels of the first double sense implication). The last part of Property (i) follows from the antisymmetric property of the commutator $\left[A_{i}, A_{j}\right]=\left[A_{j}, A_{i}\right]=0 ; \forall A_{i}, A_{j} \in \mathbf{A}_{C}$ what implies $A_{i} \in \mathbf{A}_{C} ; \forall i \in \bar{p} \Leftrightarrow v\left(A_{i}\right) \in$ $\bigcap_{i+1 \leq j \leq p} \operatorname{Ker}\left(A_{j} \oplus\left(-A_{j}^{T}\right)\right) ; \forall A_{i}, A_{j} \in \mathbf{A}_{C}$.

(ii) It follows from its equivalence with Property (i) since $\operatorname{Ker} N_{i}\left(\mathbf{A}_{C}\right) \equiv$ $\bigcap_{j(\neq i) \in \bar{p}} \operatorname{Ker}\left(A_{j} \oplus\left(-A_{j}^{T}\right)\right)$.

(iii) Property (iii) is similar to Property (i) for the whose set $M_{\mathbf{A}_{C}}$ of matrices which commute with the set $\mathbf{A}_{C}$ so that it contains $\mathbf{A}_{C}$ and, furthermore, $\operatorname{Ker} N\left(\mathbf{A}_{C}\right) \equiv \bigcap_{i \in \bar{p}} \operatorname{Ker}\left(A_{i} \oplus\right.$ $\left.\left(-A_{i}^{T}\right)\right)$.

(iv) It follows from $\bigcup_{j \in \bar{p}} \operatorname{Im}\left(A_{j} \oplus\left(-A_{j}^{T}\right)\right)=\overline{\bigcap_{j \in \bar{p}} \operatorname{Ker}\left(A_{j} \oplus\left(-A_{j}^{T}\right)\right)} ; \quad A_{j} \in \mathbf{A}_{C}$ and $\mathbf{R}^{n^{2}} \ni$ $0 \in \operatorname{Ker}\left(A_{j} \oplus\left(-A_{j}^{T}\right)\right) \cap \operatorname{Im}\left(A_{j} \oplus\left(-A_{j}^{T}\right)\right)$ but $\mathbf{R}^{n \times n} \ni X=0$ commutes with any matrix in $\mathbf{R}^{n \times n}$ so that $\mathbf{R}^{n \times n} \ni 0 \in \mathrm{MC}_{\mathbf{A}_{C}} \Leftrightarrow \mathbf{R}^{n \times n} \ni 0 \notin \overline{\mathrm{MC}_{\mathbf{A}_{C}}}$ for any given $\mathbf{A}_{C}$. commute.

(v) and (vi) are similar to (ii)-(iv) except that the members of $\mathbf{A}$ do not necessarily

Proof of Proposition 3.2. It is a direct consequence from Proposition 3.1(i)-(ii) since the existence of nonzero pair wise commuting matrices (all the members of $\mathbf{A}_{C}$ ) implies that the above matrices $N\left(\mathbf{A}_{C}\right), N_{i}\left(\mathbf{A}_{C}\right), A_{j} \oplus\left(-A_{j}^{T}\right)$ are all rank defective and have at least identical number of rows than that of columns. Therefore, the square matrices $N^{T}\left(\mathbf{A}_{C}\right) N\left(\mathbf{A}_{C}\right), N_{i}^{T}\left(\mathbf{A}_{C}\right) N_{i}\left(\mathbf{A}_{C}\right)$, and $A_{j} \oplus\left(-A_{j}^{T}\right)$ are all singular.

Proof of Theorem 3.3. (i) Any nonzero matrix $\Lambda=\operatorname{diag}(\lambda \lambda \cdots \lambda), \lambda(\neq 0) \in \mathbf{R}$ is such that $\Lambda(\neq 0) \in C_{A_{i}}(\forall i \in \bar{p})$ so that $\Lambda \in C_{\mathbf{A}}$. Thus, $0 \neq v(\Lambda) \in \operatorname{Ker} N(\mathbf{A}) \Leftrightarrow n^{2}>\operatorname{rank} N(\mathbf{A}) \geq$ $\operatorname{rank} N_{i}(\mathrm{~A}) \geq \operatorname{rank}\left(A_{i} \oplus\left(-A_{i}\right)\right) ; \forall i \in \bar{p}$ and any given set $\mathbf{A}$. Property (i) has been proved.

(ii) The first part follows by contradiction. Assume $\bigcap_{i \in \bar{p}} \operatorname{Ker}\left(A_{i} \oplus\left(-A_{i}^{T}\right)\right)=\{0\}$ then $0 \neq v(\Lambda) \notin \operatorname{Ker} N(\mathbf{A})$ so that $\Lambda=\operatorname{diag}(\lambda \quad \lambda \cdots \lambda) \notin C_{\mathbf{A}}$, for any $\lambda(\neq 0) \in \mathbf{R}$ what contradicts (i). Also, $X \in C_{A_{i}} \Leftrightarrow v(X) \in \operatorname{Ker}\left(A_{i} \oplus\left(-A_{i}^{T}\right)\right) ; \forall i \in \bar{p}$ so that $X \in C_{\mathbf{A}} \Leftrightarrow v(X) \in \bigcap_{i \in \bar{p}} \operatorname{Ker}\left(A_{i} \oplus\left(-A_{i}^{T}\right)\right)$ what is equivalent to its contrapositive logic proposition $X \in \bar{C}_{\mathbf{A}} \Leftrightarrow v(X) \in \bigcup_{i \in \bar{p}} \operatorname{Im}\left(A_{i} \oplus\right.$ $\left.\left(-A_{i}^{T}\right)\right)$.

(iii) Let $\mathbf{A}=\mathbf{A}_{C} \Leftrightarrow A_{i} \in C_{A_{j}} ; \forall j(\neq i) \in \bar{p}, \forall i \in \bar{p} \Leftrightarrow A_{i} \in C_{A_{j}} ; \forall j, i \in \bar{p}$ since $A_{i} \in$ $C_{A_{i}} ; \forall i \in \bar{p}$

$$
v\left(A_{i}\right) \in \bigcap_{i \in \bar{p}} \operatorname{Ker}\left(A_{j} \oplus\left(-A_{j}^{T}\right)\right) ; \quad \forall i \in \bar{p} \Longleftrightarrow v\left(A_{i}\right) \in \bigcap_{i \in \bar{p} \backslash\{i\}} \operatorname{Ker}\left(A_{j} \oplus\left(-A_{j}^{T}\right)\right) ; \quad \forall i \in \bar{p}
$$


On the other hand,

$$
\left(v\left(A_{i}\right) \in \bigcap_{j \in \bar{p} \backslash \bar{i}} \operatorname{Ker}\left(A_{j} \oplus\left(-A_{j}^{T}\right)\right) \Longleftrightarrow v\left(A_{i}\right) \in C_{A_{j}} ; \forall j \in \bar{p}\right) \quad \text { for any } i(<p) \in \bar{p} \text {. }
$$

This assumption implies directly that

$$
v\left(A_{i}\right) \in C_{A_{j}} ; \quad \forall j \in \bar{p} \wedge v\left(A_{i+1}\right) \in \bigcap_{j \in \overline{i+1}} C_{A_{j}} \text { for any } i(<p) \in \bar{p}
$$

which together with $v\left(A_{i+1}\right) \in \bigcap_{j \in \bar{p} \backslash \overline{i+1}} \operatorname{Ker}\left(A_{j} \oplus\left(-A_{j}^{T}\right)\right)$ implies that

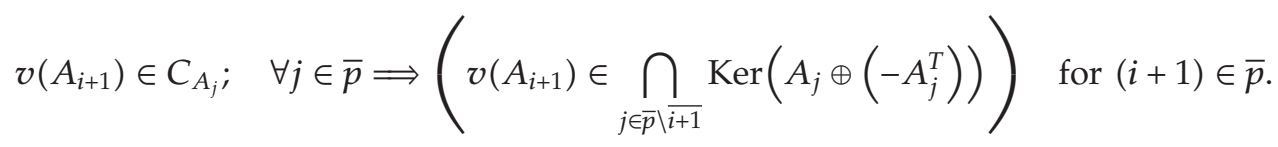

Thus, it follows by complete induction that $\mathbf{A}=\mathbf{A}_{C} \Leftrightarrow v\left(A_{i}\right) \in \bigcap_{i \in \bar{p} \backslash\{i\}} \operatorname{Ker}\left(A_{j} \oplus\left(-A_{j}^{T}\right)\right) ; \forall i \in \bar{p}$ and Property (iii) has been proved.

(iv) The definition of $\mathbf{M A}_{C}$ follows from Property (iii) in order to guarantee that $\left[X, A_{i}\right]=0 ; \forall A_{i} \in \mathbf{A}$. The fact that such a set contains properly $\mathbf{A}_{C} \cup\{0\}$ follows directly from $\mathbf{R}^{n \times n} \ni \Lambda=\operatorname{diag}(\lambda \quad \lambda \cdots \lambda)\left(\in \mathrm{MC}_{\mathbf{A}_{C}}\right) \neq \mathbf{A}_{C} \cup\{0\}$ for any $\mathbf{R} \ni \lambda \neq 0$.

Proof of Theorem 3.4. If $A_{i} \sim J_{A_{i}}=P_{i}^{-1} A_{i} P_{i}$, with $J_{A_{i}}$ being the Jordan canonical form of $A_{i}$ then $A_{i} \oplus\left(-A_{i}^{T}\right) \sim J_{A_{i}} \oplus\left(-J_{A_{i}}^{T}\right)=T_{i}^{-1}\left(A_{i} \oplus\left(-A_{i}^{T}\right)\right) T_{i}$ with $T_{i}=P_{i} \otimes P_{i}^{T} \in \mathbf{R}^{n^{2}} \times \mathbf{R}^{n^{2}}$ (see proof of Theorem 2.3) being nonsingular; $\forall i \in \bar{p}$. Thus, $\left(A_{i} \oplus\left(-A_{i}^{T}\right)\right)=T_{i}\left(J_{A_{i}} \oplus\left(-J_{A_{i}}^{T}\right)\right) T_{i}^{-1}$ so that:

$$
\begin{aligned}
N(\mathbf{A}) & =\left[\begin{array}{llll}
A_{1}^{T} \oplus\left(-A_{1}\right) & A_{2}^{T} \oplus\left(-A_{2}\right) & \cdots & A_{p}^{T} \oplus\left(-A_{p}\right)
\end{array}\right]^{T} \\
& =\left[\begin{array}{ll}
I_{p n^{2}} & -U
\end{array}\right]\left[\begin{array}{ll}
W_{1}^{T} & W_{2}^{T}
\end{array}\right]^{T}=\mathbf{T J T}_{\mathbf{a}},
\end{aligned}
$$

where

$$
\begin{aligned}
\mathbf{T} & :=\text { Block Diag }\left[\begin{array}{llll}
T_{1} & T_{2} & \cdots & T_{p}
\end{array}\right] \in \mathbf{R}^{p n^{2} \times p n^{2}}, \\
\mathbf{T}_{\mathbf{a}} & :=\left[\begin{array}{llll}
T_{1}^{-T} & T_{2}^{-T} & \cdots & T_{p}^{-T}
\end{array}\right]^{T} \in \mathbf{R}^{p n^{2} \times n^{2}}, \\
\mathbf{J} & :=\text { Block Diag }\left[\begin{array}{llll}
J_{A_{1}} \oplus\left(-J_{A_{1}}^{T}\right) & J_{A_{2}} \oplus\left(-J_{A_{2}}^{T}\right) & \cdots & J_{A_{p}} \oplus\left(-J_{A_{p}}^{T}\right)
\end{array}\right] \in \mathbf{R}^{p n^{2} \times p n^{2}} .
\end{aligned}
$$


Then,

$$
\begin{aligned}
\operatorname{Ker} N(\mathbf{A}) & =\bigcap_{i=1}^{p} \operatorname{Ker}\left[\left(A_{i} \oplus\left(-A_{i}^{T}\right)\right)\right]=\operatorname{Ker}\left(\mathbf{J T}_{\mathbf{a}}\right) \\
& \equiv \bigcap_{i=1}^{p}\left(\operatorname{Ker}\left[\left(J_{A_{i}} \oplus\left(-J_{A_{i}}^{T}\right)\right)\left(P_{i}^{-1} \otimes P_{i}^{-T}\right)\right]\right)
\end{aligned}
$$

since $\mathbf{T}$ is nonsingular. Thus, $\forall X \in \operatorname{Dom}(\mathbf{A}) \subset \mathbf{R}^{n^{2}}$ :

$$
\begin{aligned}
X \in C_{\mathbf{A}} & \Longleftrightarrow v(X) \in \operatorname{Ker} N(\mathbf{A}) \\
& \Longleftrightarrow v(X) \in\left(\bigcap_{i=1}^{p} \operatorname{Ker}\left[\left(A_{i} \oplus\left(-A_{i}^{T}\right)\right)\right]\right) \\
& \Longleftrightarrow v(X) \in\left(\bigcap_{i=1}^{p} \operatorname{Ker}\left[\left(J_{A_{i}} \oplus\left(-J_{A_{i}}^{T}\right)\right)\left(P_{i}^{-1} \otimes P_{i}^{-T}\right)\right]\right) \\
& \Longleftrightarrow v(X) \in \operatorname{Im}\left(\left(P_{i} \otimes P_{i}^{-1}\right)\left(\operatorname{Ker}\left(J_{A_{i}} \oplus\left(-J_{A_{i}}^{T}\right)\right)\right)\right) ; \quad \forall i \in \bar{p} \\
& \Longleftrightarrow v(X) \in \bigcap_{i=1}^{p}\left(\operatorname{Im}\left(\left(P_{i} \otimes P_{i}^{-1}\right)(Y)\right)\right) \\
& \Longleftrightarrow v(X) \in \bigcap_{i=1}^{p}\left(\operatorname{Im}\left(\left(P_{i} \otimes P_{i}^{-1}\right)\left(Y_{i}\right)\right)\right),
\end{aligned}
$$

where $Y_{i} \in \operatorname{Ker}\left(J_{A_{i}} \oplus\left(-J_{A_{i}}^{T}\right)\right) ; \forall i \in \bar{p}$ and $Y \in\left(\bigcap_{i=1}^{p}\left(\operatorname{Ker}\left(J_{A_{i}} \oplus\left(-J_{A_{i}}^{T}\right)\right)\right)\right)$. Property (i) has been proved. The first inequality of Property (ii) follows directly from Property (i). The results of equalities and inequalities in the second line of Property (ii) follow by the first inequality by taking into account Theorem 2.3. Property (iii) follows from the proved equivalent definitions of $C_{\mathrm{A}}$ in Property (i) by taking into account that $\left[A_{j}, A_{j}\right]=0 ; \forall j \in \bar{p}$ so that

$$
\begin{aligned}
v\left(A_{j}\right) & \in \bigcap_{i=1}^{p}\left(\operatorname{Ker}\left[\left(J_{A_{i}} \oplus\left(-J_{A_{i}}^{T}\right)\right)\left(P_{i}^{-1} \otimes P_{i}^{-T}\right)\right]\right) \\
& \Longleftrightarrow v\left(A_{j}\right) \in \bigcap_{i(\neq j)=1}^{p}\left(\operatorname{Ker}\left[\left(J_{A_{i}} \oplus\left(-J_{A_{i}}^{T}\right)\right)\left(P_{i}^{-1} \otimes P_{i}^{-T}\right)\right]\right) ; \quad \forall j \in \bar{p} .
\end{aligned}
$$




\section{Proofs of the Results of Section 4}

Proofs of Propositions 4.1-4.3

Proposition 4.1 follows by inspection of (4.1). Proposition 4.2 implies that Proposition 4.1 holds with the four involved commutators being zero. Then the left condition of Proposition 4.2 implies that $B \in C_{A}$, from Proposition 4.1, so that Proposition 4.2 holds. Proposition 4.3 is equivalent to Proposition 4.2.

Proof of Theorem 4.4. (i) Equation (4.2) is a rearrangement in an equivalent algebraic system of Proposition 4.1 in the unknown $v\left(B_{\mathrm{im}}\right)$ for given $A$ and $B_{\text {re. }}$. The system is compatible if (4.2) holds from the Kronecker-Capelli theorem. The proof of Property (ii) is similar to that of (i) with the appropriate interchange of roles of $B_{\text {re }}$ and $B_{\text {im }}$.

(iii) Since

$$
\operatorname{rank}\left[\begin{array}{c}
A_{\mathrm{im}} \oplus\left(-A_{\mathrm{im}}^{T}\right) \\
A_{\mathrm{re}} \oplus\left(-A_{\mathrm{re}}^{T}\right)
\end{array}\right]<n^{2}
$$

from Theorem 3.3(i) then $0 \neq B=B_{\text {re }} \in C_{A}$ if and only if $B_{\mathrm{re}} \in C_{A_{\mathrm{re}}} \cap C_{A_{\mathrm{im}}}(\neq \emptyset) \subset C_{A}$. The same proof follows for $0 \neq B=B_{\mathrm{im}} \in C_{A}$ since

$$
\operatorname{rank}\left[\begin{array}{c}
A_{\mathrm{re}} \oplus\left(-A_{\mathrm{re}}^{T}\right) \\
A_{\mathrm{im}} \oplus\left(-A_{\mathrm{im}}^{T}\right)
\end{array}\right]=\operatorname{rank}\left[\begin{array}{c}
A_{\mathrm{im}} \oplus\left(-A_{\mathrm{im}}^{T}\right) \\
A_{\mathrm{re}} \oplus\left(-A_{\mathrm{re}}^{T}\right)
\end{array}\right]<n^{2} .
$$

Proof of Theorem 4.5. (i) It follows in the same way as that of Theorem 4.4 by rewriting the algebraic system (4.3) in the form (4.5) which has nonzero solutions if (4.8) holds. But (4.8) always holds since $B=A \in C_{A} \cap \mathbf{C}^{n \times n}$ is nonzero if $A$ is nonzero and if $A=0 \in \mathbf{C}^{n \times n}$ then $C_{A}=\mathrm{C}^{n \times n}$.

(ii) Direct calculations yield the equivalence of (4.5) with the separation into real and imaginary parts of the subsequent algebraic system:

$$
\left(A \otimes I_{n}-I_{n} \otimes A^{*}\right) v(B)=\left[\left(A_{\mathrm{re}}+\mathbf{i} A_{\mathrm{im}}\right) \otimes I_{n}-I_{n} \otimes\left(A_{\mathrm{re}}^{T}-\mathbf{i} A_{\mathrm{im}}^{T}\right)\right]\left(v\left(B_{\mathrm{re}}\right)+\mathbf{i} v\left(B_{\mathrm{im}}\right)\right)=0
$$

which is always solvable with a nonzero solution (i.e., compatible) $\operatorname{since} \operatorname{rank}\left(A \otimes I_{n}-I_{n} \otimes A^{*}\right)<$ $n^{2}$ (otherwise, $A(\neq 0) \in C_{A}$ ).

\section{Outline of Proof of Theorem 4.6}

(i) It is a direct extension of Theorem 4.5 by decomposing the involved complex matrices in their real and imaginary parts since from Theorem 3.3(i) both left block matrices in the coefficient matrix of (4.11) have rank less than $n^{2}$. As a result, such a coefficient matrix has rank less than $2 n^{2}$ so that nonzero solutions exists to the algebraically compatible system of linear equations (4.11). As a result, a nonzero $n$-square complex commuting matrix exists. 
(ii) It is close to that of (i) but the rank condition for compatibility of the algebraic system is not needed since the coefficient matrix of (4.11) is rank defective since $A_{j} \in \mathbf{A}_{C} \Leftrightarrow$ $\left(v^{T}\left(A_{j \mathrm{re}}\right), v^{T}\left(A_{j \mathrm{im}}\right)\right)^{T}$ is in the null space of the coefficient matrix; $\forall j \in \bar{p}$.

(iii) Its proof is close to that of Theorem 4.5(ii) and it is then omitted.

Proof of Theorem 4.8. For any $B \in C_{A} \cap C^{n \times n}$ :

$$
\begin{aligned}
{[A, B] } & =0 \Longrightarrow\left(\lambda I_{n}-B\right) A=A\left(\lambda I_{n}-B\right) ; \quad \forall \lambda \in \mathbf{C} \\
& \Longrightarrow\left(\lambda I_{n}-B\right)^{-1} A=A\left(\lambda I_{n}-B\right)^{-1} ; \quad \forall \lambda \in \mathbf{C} \cap \overline{\sigma(B)} \\
& \Longrightarrow[A, f(B)]=A\left[\frac{1}{2 \pi \mathbf{i}} \oint_{C} f(\lambda)\left(\lambda I_{n}-B\right)^{-1} d \lambda\right]=\frac{1}{2 \pi \mathbf{i}} \oint_{C} f(\lambda)\left(\lambda I_{n}-B\right)^{-1} A d \lambda \\
& =\left[\frac{1}{2 \pi \mathbf{i}} \oint_{C} f(\lambda)\left(\lambda I_{n}-B\right)^{-1} d \lambda\right] A=[f(B), A]=0,
\end{aligned}
$$

where $C$ is the boundary of $D$ and consists in a set of closed rectifiable Jordan curves which contains no point of $\sigma(A)$ since $\lambda \in \mathrm{C} \cap \overline{\sigma(A)}$ so that the identity $\left(\lambda I_{n}-B\right)^{-1} A=A\left(\lambda I_{n}-B\right)^{-1}$ is true. Then, $f(B) \in C_{A} \cap C^{n \times n}$ has been proved. From Theorem 4.5, this is equivalent to $v(f(B)) \in \operatorname{Ker}\left(A \oplus\left(-A^{*}\right)\right)$.

Proof of Theorem 4.13. $B \in C_{A} \Leftrightarrow F[A, B] G=0 ; \forall F, G \in C^{n \times n}$ being nonsingular. By choosing $F^{-1}=G=T$, it follows that

$$
T^{-1}[A, B] T=T^{-1} A\left(T T^{-1}\right) B T-T^{-1} B\left(T T^{-1}\right) A T=\left[\Lambda_{A}, \Lambda_{B}\right]=0 .
$$

\section{Acknowledgments}

The author is grateful to the Spanish Ministry of Education by its partial support of this work through Grant DPI2006-00714. He is also grateful to the Basque Government by its support through Grants GIC07143-IT-269-07 and SAIOTEK S-PE08UN15. Finally, he is grateful to the reviewers by their interesting suggestions.

\section{References}

[1] S.-C. Pei, J.-J. Ding, W.-L. Hsue, and K.-W. Chang, "Generalized commuting matrices and their eigenvectors for DFTs, offset DFTs, and other periodic operations," IEEE Transactions on Signal Processing, vol. 56, no. 8, pp. 3891-3904, 2008.

[2] A. S. Phani, "On the necessary and sufficient conditions for the existence of classical normal modes in damped linear dynamic systems," Journal of Sound and Vibration, vol. 264, no. 3, pp. 741-745, 2003.

[3] B. S. Shastry, "A class of parameter-dependent commuting matrices," Journal of Physics A, vol. 38, no. 23, pp. L431-L437, 2005.

[4] R. Bhatia and L. Elsner, "On joint eigenvalues of commuting matrices," Canadian Mathematical Bulletin, vol. 39 , no. 2 , pp. $164-168,1996$.

[5] A. R. Bernstein, "Almost eigenvectors for almost commuting matrices," SIAM Journal on Applied Mathematics, vol. 21, pp. 232-235, 1971. 
[6] I. D. Berg and K. R. Davidson, "Almost commuting matrices and the Brown-Douglas-Fillmore theorem," Bulletin of the American Mathematical Society, vol. 16, no. 1, pp. 97-100, 1987.

[7] M. De la Sen, "Stability criteria for linear time-invariant systems with point delays based on onedimensional Routh-Hurwitz tests," Applied Mathematics and Computation, vol. 187, no. 2, pp. 1199_ 1207, 2007.

[8] L. A. Zadeh, Linear Systems Theory. The State Space Approach, McGraw- Hill, New York, NY, USA, 1963.

[9] M. De la Sen and N. Luo, "On the uniform exponential stability of a wide class of linear time-delay systems," Journal of Mathematical Analysis and Applications, vol. 289, no. 2, pp. 456-476, 2004.

[10] M. De la Sen and A. Ibeas, "On the global asymptotic stability of switched linear time-varying systems with constant point delays," Discrete Dynamics in Nature and Society, vol. 2008, Article ID 231710, 31 pages, 2008.

[11] M. De la Sen and A. Ibeas, "Stability results for switched linear systems with constant discrete delays," Mathematical Problems in Engineering, vol. 2008, Article ID 543145, 28 pages, 2008.

[12] S. Kim, S. A. Campbell, and X. Liu, "Delay independent stability of linear switching systems with time delay," Journal of Mathematical Analysis and Applications, vol. 339, no. 2, pp. 785-801, 2008.

[13] D. Liu, X. Liu, and S. Zhong, "Delay-dependent robust stability and control synthesis for uncertain switched neutral systems with mixed delays," Applied Mathematics and Computation, vol. 202, no. 2, pp. 828-839, 2008.

[14] M. De la Sen and A. Ibeas, "Stability results of a class of hybrid systems under switched continuoustime and discrete-time control," Discrete Dynamics in Nature and Society, vol. 2009, Article ID 315713, 28 pages, 2009.

[15] A. Ibeas and M. De la Sen, "Exponential stability of simultaneously triangularizable switched systems with explicit calculation of a common Lyapunov function," Applied Mathematics Letters, vol. 22, no. 10, pp. 1549-1555, 2009.

[16] S. Akbari, F. Moazami, and A. Mohammadian, "Commutativity of the adjacency matrices of graphs," Discrete Mathematics, vol. 309, no. 3, pp. 595-600, 2009.

[17] B. Santhanam and T. S. Santhanam, "On discrete Gauss-Hermite functions and eigenvectors of the discrete Fourier transform," Signal Processing, vol. 88, no. 11, pp. 2738-2746, 2008.

[18] S.-C. Pei, W.-L. Hsue, and J.-J. Ding, "DFT-commuting matrix with arbitrary or infinite order second derivative approximation," IEEE Transactions on Signal Processing, vol. 57, no. 1, pp. 390-394, 2009.

[19] E. Callegari and M. Ghisi, “Local $C^{1}$ solutions to some non-linear PDE system," Mathematical Methods in the Applied Sciences, vol. 29, no. 10, pp. 1107-1119, 2006.

[20] M. Yavari and A. R. Ashrafi, "A new method for computing the symmetry of big fullerene C180," Asian Journal of Chemistry, vol. 20, no. 7, pp. 5119-5122, 2008.

[21] O. M. Baksalary and P. Kik, "On commutativity of projectors," Linear Algebra and Its Applications, vol. 417, no. 1, pp. 31-41, 2006.

[22] G. H. Shortley and G. E. Kimball, "Analysis of non-commuting vectors with applications to quantum mechanics and vector calculus," Proceedings of the National Academy of Sciences of the United States of America, vol. 20, no. 1, pp. 82-84, 1934.

[23] D. Liberzon, Switching in Systems and Control, Systems \& Control: Foundations and Applications, Birkhäuser, Boston, Mass, USA, 2003.

[24] Z. Li, Y. Soh, and C. Wen, Switched and Impulsive Systems: Analysis, Design and Applications, vol. 313 of Lecture Notes in Control and Information Sciences, Springer, Berlin, Germany, 2005.

[25] Z. Sun and S. S. Ge, Switched Linear Systems: Control and Design, Springer, London, UK, 2005.

[26] M. De la Sen, "Adaptive stabilization of continuous-time systems through a controllable modified estimation model," Mathematical Problems in Engineering, no. 2, pp. 109-131, 2004.

[27] J. Zhao and D. J. Hill, "On stability, $L_{2}$-gain and $H_{\infty}$ control for switched systems," Automatica, vol. 44, no. 5, pp. 1220-1232, 2008.

[28] H. Lin and P. J. Antsaklis, "Stability and stabilizability of switched linear systems: a survey of recent results," IEEE Transactions on Automatic Control, vol. 54, no. 2, pp. 308-322, 2009.

[29] H. Yang, G. Xie, T. Chu, and L. Wang, "Commuting and stable feedback design for switched linear systems," Nonlinear Analysis: Theory, Methods \& Applications, vol. 64, no. 2, pp. 197-216, 2006.

[30] H. Ishii and B. A. Francis, "Stabilizing a linear system by switching control with dwell time," in Proceedings of the American Control Conference (ACC'01), pp. 1876-1881, Arlington, Va, USA, 2001.

[31] Z. Wu and F. Ben Amara, "Parameterized regulator synthesis for bimodal linear systems based on bilinear matrix inequalities," Mathematical Problems in Engineering, vol. 2008, Article ID 341720, 22 pages, 2008. 
[32] A. Leonessa, W. M. Haddad, and V. Chellaboina, "Nonlinear robust hierarchical control for nonlinear uncertain systems," Mathematical Problems in Engineering, vol. 5, no. 6, pp. 499-542, 2000.

[33] M. Margaliot and D. Liberzon, "Lie-algebraic stability conditions for nonlinear switched systems and differential inclusions," Systems \& Control Letters, vol. 55, no. 1, pp. 8-16, 2006.

[34] M. De la Sen, "Robust adaptive control of linear time-delay systems with point time-varying delays via multiestimation," Applied Mathematical Modelling, vol. 33, no. 2, pp. 959-977, 2009.

[35] M. De la Sen and A. Ibeas, "On the stability properties of linear dynamic time-varying unforced systems involving switches between parameterizations from topologic considerations via graph theory," Discrete Applied Mathematics, vol. 155, no. 1, pp. 7-25, 2007.

[36] A. Bilbao-Guillerna, M. De la Sen, S. Alonso-Quesada, and A. Ibeas, "A stable multimodel scheme control for the regulation of the transient behavior of a tunnel-diode trigger circuit," ISA Transactions, vol. 46, no. 3, pp. 313-326, 2007.

[37] K. S. Narendra and J. Balakrishnan, "Adaptive control using multiple models," IEEE Transactions on Automatic Control, vol. 42, no. 2, pp. 171-187, 1997.

[38] K. S. Narendra and J. Balakrishnan, "Improving transient response of adaptive control systems using multiple models and switching," IEEE Transactions on Automatic Control, vol. 39, no. 9, pp. 1861-1866, 1994.

[39] A. Ibeas and M. De la Sen, "Robustly stable adaptive control of a tandem of master-slave robotic manipulators with force reflection by using a multiestimation scheme," IEEE Transactions on Systems, Man, and Cybernetics Part B, vol. 36, no. 5, pp. 1162-1179, 2006.

[40] Z. Sun and S. S. Ge, "Analysis and synthesis of switched linear control systems," Automatica, vol. 41, no. 2, pp. 181-195, 2005.

[41] Z. Sun, "A robust stabilizing law for switched linear systems," International Journal of Control, vol. 77, no. 4, pp. 389-398, 2004.

[42] S. Gasiorowicz, Quantum Physics, John Wiley \& Sons, New York, NY, USA, 1974.

[43] J.-L. Basdevant, Lectures on Quantum Mechanics, Springer, New York, NY, USA, 2007. 


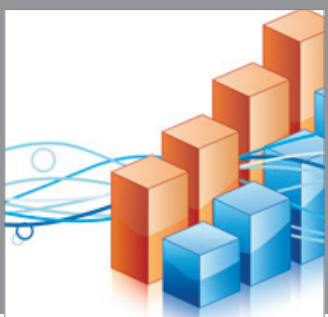

Advances in

Operations Research

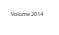

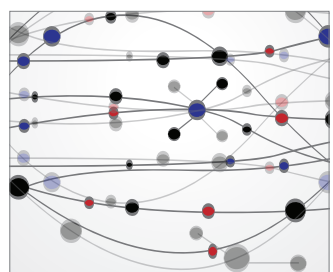

\section{The Scientific} World Journal
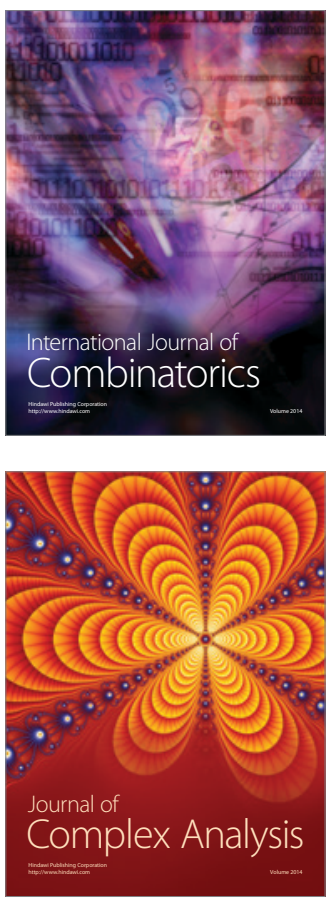

International Journal of

Mathematics and

Mathematical

Sciences
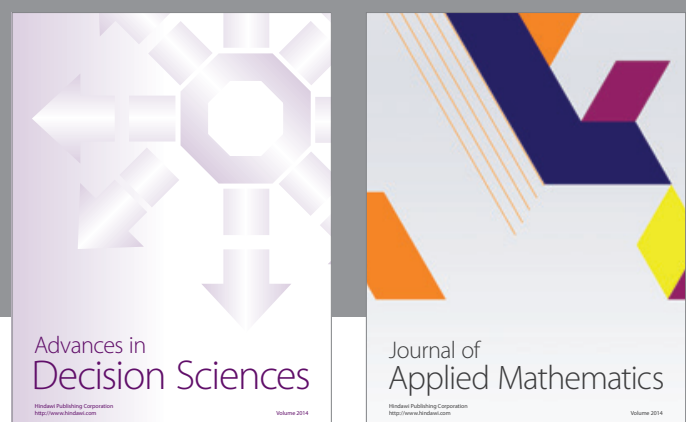

Journal of

Applied Mathematics
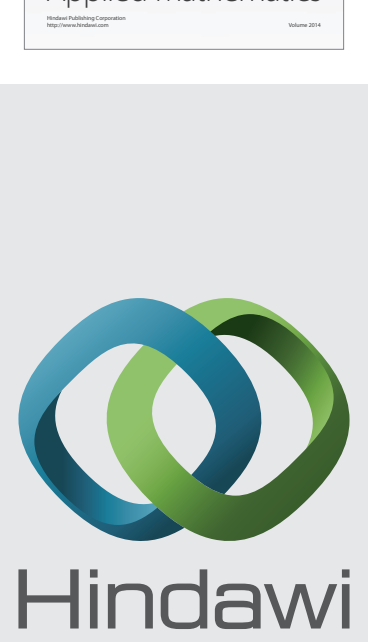

Submit your manuscripts at http://www.hindawi.com
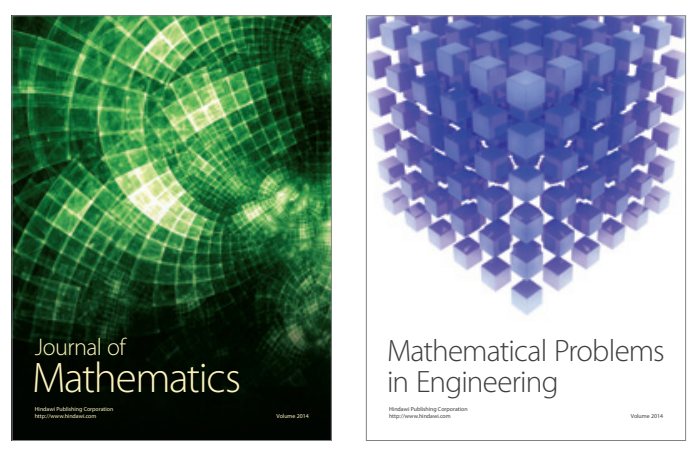

Mathematical Problems in Engineering
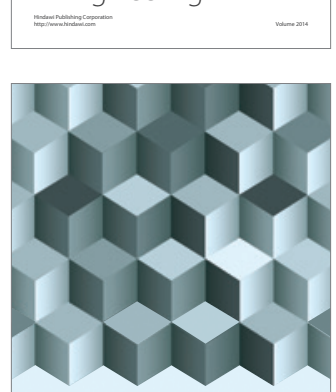

Journal of

Function Spaces
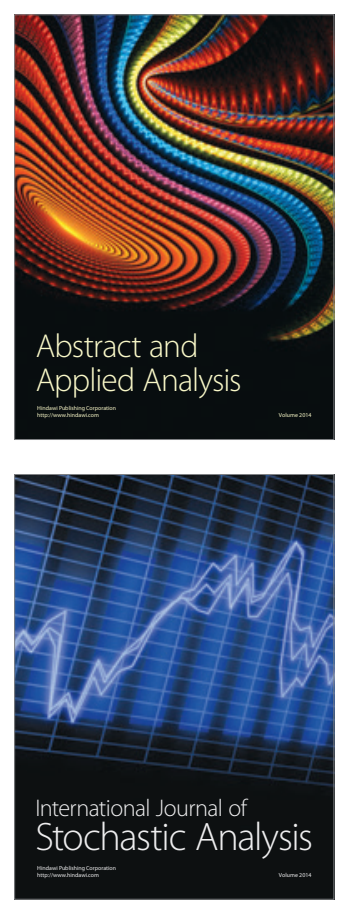

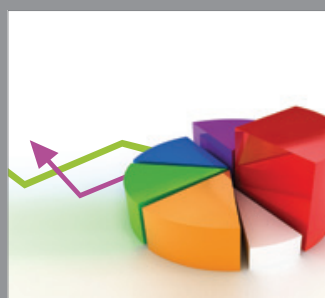

ournal of

Probability and Statistics

Promensencen
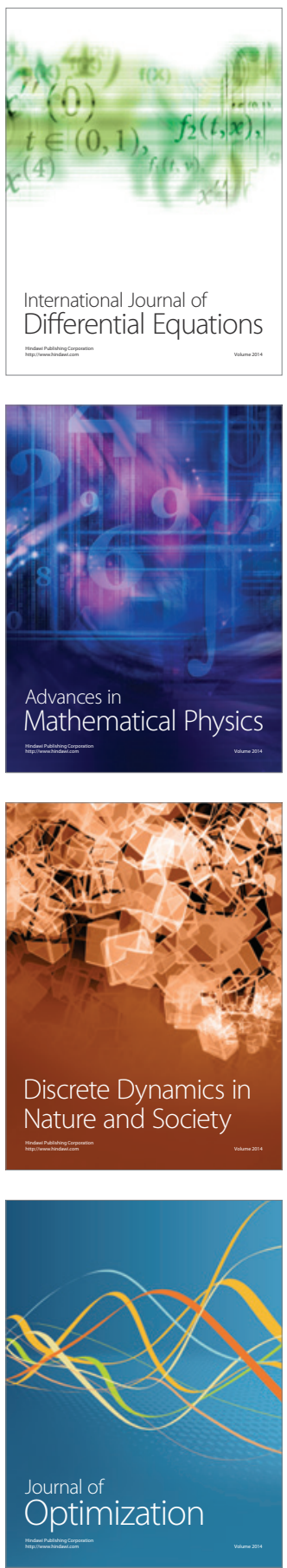